\title{
GRID-Independent molecular descriptor analysis and molecular docking studies to mimic the binding hypothesis of Y-Aminobutyric Acid Transporter 1 (GAT1) inhibitors
}

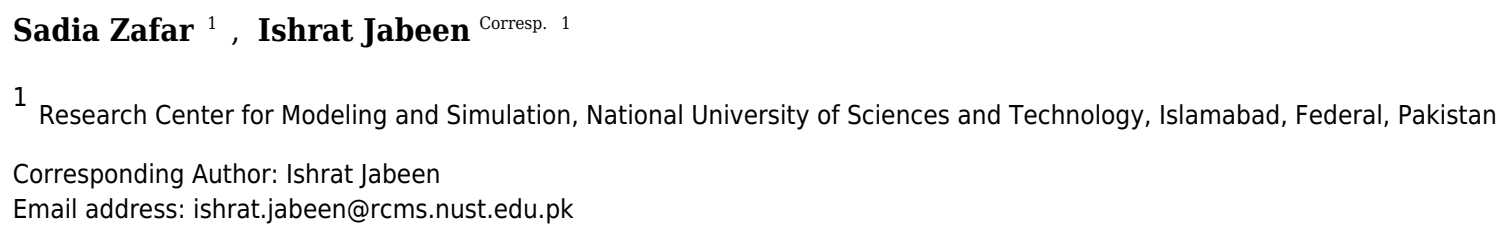

Background: The $\gamma$-aminobutyric acid (GABA) transporter GAT1 is involved in GABA transport across the biological membrane in and out of the synaptic cleft. The efficiency of this $\mathrm{Na}^{+}$coupled GABA transport is regulated by an electrochemical gradient, which is directed inward under normal conditions. However, in certain pathophysiological situations, including strong depolarization or an imbalance in ion homeostasis, the GABA influx into the cytoplasm is increased by re-uptake transport mechanism. This mechanism may lead to extra removal of extracellular GABA which results in numerous neurological disorders such as epilepsy. Thus, small molecule inhibitors of GABA re-uptake may enhance GABA activity at the synaptic clefts. Methods: In the present study, various GRID Independent Molecular Descriptor (GRIND) models have been developed to shed light on the 3D structural features of hGAT1 inhibitors using nipecotic acid and N-diarylalkenyl piperidine analogs. Further, a binding hypothesis has been developed for the selected GAT1 antagonists by molecular docking inside the binding cavity of human GAT1 (hGAT1) homology model. Results: Our results indicate that two hydrogen bond acceptors, one hydrogen bond donor and one hydrophobic region at certain distances from each other play an important role in achieving high inhibitory potency against hGAT1. Our docking results elucidate the importance of the $\mathrm{COOH}$ group in hGAT1 antagonists by considering substitution of the $\mathrm{COOH}$ group with an isoxazol ring in compound 37, which subsequently leads to a three order of magnitude decrease in biological activity of $\mathbf{3 7}\left(\mathrm{IC}_{50}=38 \mu \mathrm{M}\right)$ as compared to compound $\mathbf{1}\left(\mathrm{IC}_{50}=0.040 \mu \mathrm{M}\right)$. Discussion: Our docking results are strengthened by the structure activity relationship (SAR) of the data series as well as by GRIND models, thus providing a significant structural basis for understanding the binding of antagonists, which may be useful for guiding the design of hGAT1 inhibitors. 
1 GRID-Independent molecular descriptor analysis and molecular

\section{docking studies to mimic the binding hypothesis of $\gamma$-Aminobutyric Acid Transporter 1 (GAT1) inhibitors}

Sadia Zafar ${ }^{1}$ and Ishrat Jabeen ${ }^{1 *}$

${ }^{1}$ Research Center for Modeling and Simulation (RCMS), National University of Sciences and Technology (NUST), Sector H-12, 44000, Islamabad, Pakistan

*Corresponding Author Ishrat Jabeen

Research Center for Modeling and Simulation (RCMS), National University of Sciences and Technology (NUST), Sector H-12, Islamabad, 44000, Pakistan. Email: ishrat.jabeen@rcms.nust.edu.pk

\section{Abstract}

Background: The $\gamma$-aminobutyric acid (GABA) transporter GAT1 is involved in GABA transport across the biological membrane in and out of the synaptic cleft. The efficiency of this $\mathrm{Na}^{+}$coupled GABA transport is regulated by an electrochemical gradient, which is directed inward under normal conditions. However, in certain pathophysiological situations, including strong depolarization or an imbalance in ion homeostasis, the GABA influx into the cytoplasm is increased by re-uptake transport mechanism. This mechanism may lead to extra removal of extracellular GABA which results in numerous neurological disorders such as epilepsy. Thus, small molecule inhibitors of GABA re-uptake may enhance GABA activity at the synaptic clefts. Methods: In the present study, various GRID Independent Molecular Descriptor (GRIND) models have been developed to shed light on the 3D structural features of hGAT1 inhibitors using nipecotic acid and N-diarylalkenyl piperidine analogs. Further, a binding hypothesis has been developed for the selected GAT1 antagonists by molecular docking inside the binding cavity of human GAT1 (hGAT1) homology model.

Results: Our results indicate that two hydrogen bond acceptors, one hydrogen bond donor and one hydrophobic region at certain distances from each other play an important role in achieving high inhibitory potency against hGAT1. Our docking results elucidate the importance of the $\mathrm{COOH}$ group in hGAT1 antagonists by considering substitution of the $\mathrm{COOH}$ group with an isoxazol ring in compound $\mathbf{3 7}$, which subsequently leads to a three order of magnitude decrease in biological activity of $37\left(\mathrm{IC}_{50}=38 \mu \mathrm{M}\right)$ as compared to compound $\mathbf{1}\left(\mathrm{IC}_{50}=0.040 \mu \mathrm{M}\right)$. 
33 Discussion: Our docking results are strengthened by the structure activity relationship (SAR) of

34 the data series as well as by GRIND models, thus providing a significant structural basis for

understanding the binding of antagonists, which may be useful for guiding the design of hGAT1 inhibitors.

Keywords: GABA Transporter 1 (GAT1), Tiagabine, 3D QSAR, GRIND Model, hGAT1 Inhibitors, Docking, Structure-Activity Relationship (SAR)

\section{Introduction}

Brain functioning is controlled by neuron circuits that release excitatory and inhibitory neurotransmitters like glutamate and $\gamma$-aminobutyric acid (GABA) and neuromodulators like norepinephrine, dopamine and serotonin (Heng et al., 2007). The removal of neurotransmitters from the extracellular space (i.e., between pre- and post- synaptic neurons) is regulated by their respective transporters (Calapai et al., 2001). However, imbalances in the levels of these neurotransmitters at synaptic clefts which may be associated with several neurological disorders, including Alzheimer's disease, schizophrenia, Parkinson's disease, anxiety, sleep disorders and epilepsy (Sherin and Nemeroff, 2011;Pirttimaki et al., 2013;Shetty and Bates, 2015). Most of these neurological disorders are associated with the GABAergic system and are mainly modulated using allosteric agonists of the GABAA receptor. However, inhibition of the GABA re-uptake transport to maintain its concentration gradient at synaptic clefts represents a promising concept for treating neurological disorders (Carvill et al., 2015).

GABA transporters (GATs) are categorized into four subtypes, GAT1-3, and BGT1 (betaine/GABA transporter 1) (Jin et al., 2011). GAT1 and 3 are mainly expressed at GABAergic neurons and glial cells, respectively, throughout the brain (Minelli et al., 1995;Minelli et al., 1996;Conti et al., 1998; Melone et al., 2015). However, GAT2 is localized at arachnoid and ependymal cells and has very low expression in neurons and glial cells in the brain (Conti et al., 1999;Jin et al., 2011); BGT1 is expressed, from low to high concentration, in the liver, kidney, meninges and at the blood brain barrier (Anderson et al., 2010). All GATs belong to the neurotransmitter sodium symporter (NSS) family. These transporters use a sodium gradient for re-uptake of the neurotransmitters out of the synaptic cleft; however, in certain cases a reverse transport mode is also known, which releases the neurotransmitter in a nonvesicular way (Yu et al., 1998). Overall, 75\% of GABA re-uptake is mediated by GAT1 (Parpura and Haydon, 2008;Zafar and Jabeen, 2018). This reflects that GAT1 is mainly accountable for 
64 GABA transport and related disorders. Therefore, development of potential antagonists of this 65 transporter to maintain the concentration gradient of GABA at synaptic clefts may represent a potential therapeutic strategy. Up to now, Tiagabine is the only second-generation FDA approved anticonvulsant agent that selectively inhibits Homo sapiens GAT1 (hGAT1). However, Tiagabine analogs that have been developed are often associated with off target toxicity and poor ADME-Tox properties that lead to side effects such as sedation, tremors and ataxia (Madsen et al., 2011). Thus, developing new chemical scaffolds of GABA reuptake inhibitors (i.e., hGAT1 antagonists) that have maximum efficacy and reduced toxicity might aid in the successful treatment of neuronal disorders.

Previously, various antagonists of hGAT1, including nipecotic acid, guvacine, proline, pyrrolidine, azetidine and THPO derivatives (Dalby, 2000;Andersen et al., 2001;Clausen et al., 2005;Fülep et al., 2006;Faust et al., 2010;Hellenbrand et al., 2016;Schmidt et al., 2017;Lutz et al., 2018;Tóth et al., 2018), have been synthesized and pharmacologically tested and optimized using SAR data. Additionally, several ligand-based strategies including 2D QSAR (Jurik et al., 2013), CoMFA (Zheng et al., 2006) and pharmacophore models (Hirayama et al., 2001;Nowaczyk et al., 2018) have been developed to optimize small molecule inhibitors against hGAT1. However, most of these studies were class specific, focusing on nipecotic acid derivatives (Petrera et al., 2015), Tiagabine analogs (Jurik et al., 2015) and triarylnipecotic acid derivatives (Dhar et al., 1994). Recently, a nipecotic acid derivative DDPM-2571 has been synthesized with one log unit greater inhibitory potency against GAT1 as compared to Tiagabine which showed anticonvulsant, antidepressant and antinociceptive effects in mouse models (Sałat et al., 2017). Moreover, a novel class of allosteric GAT1 antagonists has been identified through mass spectrometry screening of pseudostatic hydrazone libraries. Hauke et al., suggested that the identified allosteric nipecotic acid derivatives may provide physiological relevance in terms of hGAT1 modulation as their interaction in hGAT1 binding pocket differs from Tiagabine (Hauke et al., 2018). Additionally, some reports also suggest 5-aminolevulinic acid (5-Ala) may also inhibit the cellular uptake of GABA by GAT isoforms (Rud et al., 2000). Until very recent, no X-ray crystal structure of any hGAT has been published. Therefore, various hGAT1 models in different conformations have been developed previously using the crystal structure of the leucine transporter (LeuT) from Aquifex aeolicus (PDB ID: 3F3A) as a template. These models 
94

95

96

97

98

99

100

101

102

103

104

105

106

107

108

109

110

111

112

113

114

115

116

117

118

119

120

121

122

123

124

may assist to study the binding of hGAT1 antagonists and to study the ion dependent transport mechanistic of GABA through hGAT1 (Bicho and Grewer, 2005;Jurik et al., 2015).

In the present study, we aim to develop predictive GRIND models to provide deeper insight into the 3D structural features of hGAT1 antagonists. Moreover, a recently published Xray structure of dopamine transporter in Drosophila melanogaster (dDAT, PDB ID: 4XP4, resolution: $2.8 \AA$, sequence identity: 46\%) (Wang et al., 2015b) is used in the current study to build a model of hGAT1, followed by molecular docking studies to probe how nipecotic acid and $\mathrm{N}$-diarylalkenyl piperidine analogs bind to the binding cavity of hGAT1.

\section{Methods}

Dataset

A complete workflow of hGAT1 antagonists data preprocessing and cleaning has been provided in Fig. 1. Briefly, a dataset of 580 hGAT1 antagonists, along with their respective binding affinities $\left(\mathrm{IC}_{50}\right)$ ranging from $0.04-8511 \mu \mathrm{M}$, was obtained from the literature (Dhar et al., 1994;Schousboe, 2000;Clausen et al., 2005;Clausen et al., 2006;Fülep et al., 2006;Zheng et al., 2006;Alexander et al., 2007;Reith, 2007;Faust et al., 2010;Alexander et al., 2011;Nakada et al., 2013; Quandt et al., 2013; Sitka et al., 2013). Subsequently, duplicates and fragments were removed from the data, followed by the removal of antagonists with a molecular mass less than 150 and $\mathrm{IC}_{50}>100 \mu \mathrm{M}$. The duplicate antagonists were the replicated chemical compounds with biological activities determined through different biological assays including [3H] GABA uptake assay, GAT1 transport assay, radio-ligand binding assay and equilibrium binding assay using different expression systems like Xenopus oocytes and HEK cell lines (Dhar et al., 1994;Kragler et al., 2008;Nakada et al., 2013). Moreover, the antagonists with molecular mass less than 150 were excluded from the analysis because they were representing molecular fragments and therefore may not be selective against the hGAT1. Similarly, antagonists with $\mathrm{IC}_{50}>100 \mu \mathrm{M}$ were also discarded as they reflect least active compounds in comparison with the most active antagonist of the database $\left(\mathrm{IC}_{50}=0.040 \mu \mathrm{M}\right)$. Thus, only the data with $\mathrm{IC}_{50}$ values ranging from 0.040-100 $\mu \mathrm{M}$ was used for further analysis. Overall, our data comprises of total 215 antagonists that were further subjected to biological cleaning by selecting only those antagonists whose $\mathrm{IC}_{50}$ values were evaluated using a radio labeled [3H] GABA uptake assay (152 antagonists) in hGAT1 expressing HEK cells (102 antagonists). Furthermore, 3D structures of the selected 102 antagonists (Table S1) were constructed and energy minimized using the MMFF94x force field 
125 (Halgren, 1996) in MOE version 2013.0802 (MOE, 2013.08). The final data set of the 102 126 antagonists of hGAT1 consists of nipecotic acid, proline, pyrrolidine, exo-THPO and N127 diarylalkenyl piperidine derivatives that follow a general pattern of attachment of a $\mathrm{COOH}$ group 128 at the ortho, meta or para positions of piperidine, proline, pyrrolidine, or azetidine rings, 129 followed by a linker region of variable lengths. The general architecture of hGAT1 antagonists in 130 the present data set is represented in Fig. S1. It has been reported previously that attachment of 131 aromatic moieties to the linker region is highly correlated with the activity of hGAT1 antagonists 132 (Faust et al., 2010). Depending on the type of cyclic moieties attached at the linker region, the 133 present data set of hGAT1 antagonists was divided into three main classes (classes A, B and C). 134 Overall, the whole data set of 102 hGAT1 inhibitors in Table S1 was further divided into a 135 training set $(80 \%)$ and a test set $(20 \%)$ by using the diverse subset selection method (Schmuker 136 et al., 2004;Gillet, 2011). Briefly, 300 2D as well as 3D descriptors available in MOE version 1372013.0802 (MOE, 2013.08) were computed to obtain a distance calculation for each database 138 entry. 20\% of the data structures (19 compounds) with larger distance values from each other 139 were selected as the test set and the remaining 83 compounds (80\%) were used to train the model 140 (Minh et al., 2009) using GRIND (Durán and Pastor, 2011). Additionally, a recently published data set of 15 nipecotic acid derivatives was used as validation set (Lutz et al., 2017).

\section{GRIND}

144

145

It has been previously reported that GRIND variables are highly dependent on the 3D conformations of molecules (Durán and Pastor, 2011). Therefore, multiple conformational search approaches were used to generate four different 3D conformational sets of the training data. Subsequently, each independent sets of molecular conformations, including energy minimized, extended 3D, induced fit docking solutions, and molecular alignment conformations by pharmacophore mapping approach of hGAT1 antagonists along with their - $\log \mathrm{IC}_{50}$ values, were independently loaded into the software package Pentacle v 1.06 (Durán and Pastor, 2011) for the construction of four different GRIND models. Various authors have demonstrated that hGAT1 antagonists bind in their protonated state (Jurik et al., 2015). Therefore, all the compounds in the different conformational sets were protonated at a physiological $\mathrm{pH}$ of 7.4. A complete protocol to train the 3D QSAR model using GRID Independent molecular descriptors (GRIND) is provided in Fig. S2. Briefly, includes following steps: 
156 done using four different probes including DRY (hydrophobic probe), TIP (steric hot-spot

157

158

159

160

161

162

163

164

165

166

167

168

169

170

171

172

173

174

175

176

177

178

179

180

181

182

183

184

185

defining molecular shape), N1 (hydrogen bond acceptor) and O (hydrogen bond donor) within a molecule. The total energy at each node was calculated as a sum of the Lennard-Jones potential $\left(\mathrm{E}_{\mathrm{lj}}\right)$ (Lennard-Jones, 1931;Bouanich, 1992), the electrostatic energy $\left(\mathrm{E}_{\mathrm{el}}\right)$, and the hydrogen bond energies $\left(\mathrm{E}_{\mathrm{hb}}\right)$ by iteratively placing each probe at different GRID steps:

$$
E_{x y z}=\sum E_{l j}+\sum E_{e l}+\sum E_{h b}
$$

2. Discretization: AMANDA algorithm (Durán et al., 2008) was used to discretize the MIFs using default energy cutoff values of $-0.75,-0.5,-4.2$ and -2.6 for the TIP, DRY, N1 and O probes respectively to pre-filter the nodes that fails to meet the energy cutoffs.

3. Encoding: The consistently large auto and cross correlation (CLACC) algorithm (Durán and Pastor, 2011) with default parameters was used for the encoding of the pre-filtered nodes at each discretization step. This produces consistent sets of variables whose values are directly represented in the form of correlogram plots. To correlate the structural variance of the training set data with respective biological activity $\left(-\log \mathrm{IC}_{50}\right)$, a Partial Least Square (PLS) analysis has been performed using the full set of 570 active GRIND variables for each model. Moreover, to remove the inconsistent set of variables, cycles of Fractional Factorial Design (FFD) (Baroni et al., 1993) were employed to obtain a good statistical model.

\section{Multiple conformational analysis}

\section{Standard $3 D$ conformations}

Software CORINA v4.1.0 (Sadowski, 2003) was used to generate standard 3D conformations of the molecules. Briefly, CORINA build the 3D model of a molecule by combining the monocentric fragments with standard bond lengths, bond angles and dihedral angles, including the torsion angles of ring systems for their proper closure and to minimize the non-bonded (Van der Waals and electrostatic) interactions that occur within the flexible parts of the molecules (Sadowski et al., 2004). Finally, generated standard 3D conformation of each hGAT1 antagonist in the data set was further subjected to Pentacle v 1.06 (Durán and Pastor, 2011) for GRIND analysis.

\section{Energy minimized conformations}

The stochastic search algorithm in MOE v2013.0802 (MOE, 2013.08) was used to produce energy minimized conformations of the hGAT1 antagonists. A total of 250 
186 conformations were generated and ranked according to their energy values. Each individual in

187 the data set with the lowest energy score was selected for the GRIND analysis.

188

189

190

191

192

193

194

195

196

197

198

199

200

201

202

203

204

205

206

207

208

209

210

211

212

213

214

215

\section{Induced fit docking (IFD)}

The IFD (Sherman et al., 2006) protocol in MOE v2013.0802 (MOE, 2013.08) was followed to generate the docking solutions of 102 hGAT1 antagonists within the binding pocket of the hGAT1 model. The binding cavity was selected to include the residues already known to be involved in ligand-protein interactions in hGAT1, i.e., G59, Y60, A61, I62, G63, G65, N66, W68, Y86, L136, Y139, Y140, I143, Q291, F294, S295, Y296, G297, L300, N327, S328, S331, A358, L392, D395, S396 and D451 (Zhou et al., 2006;Skovstrup et al., 2010;Jurik et al., 2015). Overall, 20 poses per ligand were generated using default scoring function (London $\mathrm{dG}$ ) and placement method (Triangle Matcher). Finally, the best scored docking conformation for each ligand was selected for further GRIND analysis.

\section{Pharmacophore mapping approach}

Another set of molecular conformations was generated by the pharmacophore mapping approach (Martin et al., 1993) using MOE v2013.0802 (MOE, 2013.08). Briefly, the standard 3D conformation of the prototype ligand Tiagabine was used as a template for the flexible alignment of the rest of the data. The best scored aligned system based on the energy values, shown in Fig. 2, was selected to develop the GRIND model.

Overall, the relevance of structural properties to the binding affinity has been determined by Partial Least Squares (PLS) analysis. However, the value of the correlation coefficient $\left(\mathrm{R}^{2}\right)$ for the external test set validation was determined through equation 1 as described by Alexander et al (Alexander et al., 2015).

$$
R^{2}=\frac{\sum(y-\hat{y})^{2}}{\sum(y-\bar{y})^{2}}
$$

(Equation 1)

Where y represents the experimentally determined biological activity $\left(-\log \mathrm{IC}_{50}\right)$ of the data set, $\bar{y}$ is its mean value and $\hat{y}$ represent the corresponding predicted biological activity $(-\log$ $\mathrm{IC}_{50}$ ) by the GRIND model. The highly predictive final GRIND model helps to identify the 3D structural features of hGAT1 antagonists. However, in order to get deeper insight into the ligand interaction profiles within the hGAT1 binding site, further structure-based studies have been performed.

\section{Homology modeling}


Due to the absence of a crystal structure of hGAT1, comparative modeling was

217 performed using the recently solved crystal structure of the dopamine transporter in Drosophila 218 melanogaster (dDAT, resolution: $2.80 \AA$ A, PDB ID: 4XP4 ) (Wang et al., 2015a). Comparative 219 modeling of hGAT1 was performed using Modeller 9v8 software (Šali et al., 1995) Briefly, the 220 primary sequence of hGAT1 (P30531) was retrieved from the UniProt KB databank (Magrane, 2011). Homologous transporter proteins include the leucine transporter (LeuT) and the dopamine

222

223

224

225

226

227

228

229

230

231

232

233

234

235

236

237

238

239

240

241

242

243

244

245

246 transporter (DAT) in the bacteria Aquifex aeolicus (Yamashita et al., 2005) and Drosophila melanogaster, respectively (Wang et al., 2015a). Therefore, the homologous protein dDAT (PDB ID: 4XP4) in an open-to-out conformation was selected as a template for multiple sequence alignment (Schrodinger Release, 2017) because it shares 46\% sequence identity with hGAT1 as identified by BLASTp algorithm against the Protein Data Bank database (https://blast.ncbi.nlm.nih.gov/), compared to AaLeuT, which only shares $25 \%$ sequence identity with hGAT1. Additionally, dDAT also contains a $\mathrm{Cl}^{-}$ion, which is necessary to maintain the ionic concentration gradient in mammalian GABA transport (Wang et al., 2015b). In contrast, in $A a$ LeuT, the $\mathrm{Cl}^{-}$ion was not co-crystallized.

Flanking regions of the $\mathrm{N}$ - and C-terminus of hGAT1 were removed from the hGAT1 model built via Modeller 9v8 (Šali et al., 1995). However, no residues were trimmed from the extracellular loop 2 (EL2) of hGAT1, as was done in previously reported studies, due to the difference in the number of amino acid residues in EL2 for AaLeuT and hGAT1 (Baglo et al., 2013). The sodium ions and a chloride ion in a stoichiometry of 2:1 were added at the positions seen in dDAT. Briefly, 100 models of hGAT1 were built and the quality of these models was evaluated through ERRAT (Colovos and Yeates, 1993), PROCHECK (Lovell SC, 2003) and Verify3D (Bowie et al., 1991;Lüthy et al., 1992) using the web server at http://servicesn.mbi.ucla.edu/. The final model was energy minimized using the MMFF94x force field of LigX (Labute, 2008) in MOE v2013.08 (Halgren, 1996). Finally, the model quality was again evaluated using a Ramachandran plot (Ho and Brasseur, 2005).

\section{Docking and pose analysis of selected hGAT1 modulators}

Molecular docking was performed to get deeper insights into the binding of hGAT1 antagonists using GOLD suite v5.2.2 (Jones et al., 1995). To remove any bias in the pose generation step, different rotamers of the side chains were sampled. However, the ligand binding site was kept flexible. The binding site was sampled by keeping a distance of $17 \AA$ around the 
247 amino acid residues G59, Y60, A61, I62, G63, G65, N66, W68, Y86, L136, Y139, Y140, I143,

248 Q291, F294, S295, Y296, G297, L300, N327, S328, S331, A358, L392, D395, S396 and D451.

249 These amino acid residues are already known from mutagenesis data to be important in

250 interactions within hGAT1 (Zhou et al., 2006;Skovstrup et al., 2010; Jurik et al., 2015).

251 Additionally, $2 \mathrm{Na}^{+}$ions and a $\mathrm{Cl}^{-}$ion that are known for their role in the GABA transport

252 mechanism were also incorporated into the binding cavity (Zhou et al., 2006;Skovstrup et al.,

253 2010; Jurik et al., 2015). The side chains of hGAT1 (with the exception of ligand binding site

254 residues) were kept rigid while the antagonists were treated flexible by performing 100 genetic

255 algorithm runs per molecule using the gold score fitness function (Jones et al., 1995). The best

256 pose for each ligand inside the hGAT1 binding pocket was selected for further ligand-protein

257 interaction analysis.

258 To illustrate the binding hypothesis for hGAT1 antagonists with chemically different 259 scaffolds, the docking solutions of selected compounds from each class, including compounds $\mathbf{2}$, 2606,8 , and 15 from class A, compounds 14, 27, 36, and 40 from class B and compounds 1, 3, 4, 37, 261 and $\mathbf{8 4}$ from class $\mathrm{C}$ were used for further hierarchical clustering analysis based on the root mean 262 square deviation (RMSD) of the common scaffold (Stanton et al., 1999) of each respective 263 antagonist class. The compound selection criteria were solely based on the SAR data, as 264 explained in the Results section. A complete docking and pose selection protocol is provided in 265 Fig. S3. Overall, a total of 172, 110 and 64 clusters were obtained for classes A, B and C, 266 respectively, at $2 \AA$ on the basis of the RMSD of the heavy atoms of the common scaffold 267 (Stanton et al., 1999). Final cluster of binding poses from each class was further selected on the 268 basis of SAR data as explained in results section.

\section{Results}

\section{GRID Independent Molecular Descriptor (GRIND) analysis}

The statistical parameters for each GRIND model developed from different 273 conformational set are shown in Table S2. Unfortunately, none of the four conformational sets of ligands produced a statistically good model with the full set of variables. Thus, Fractional Factorial Design (FFD) (Baroni et al., 1993) was applied to reduce the number of inconsistent variables in each model. After the first cycle of FFD, the statistical parameters of each model were slightly improved. However, a final model with good statistical parameters $\left(\mathrm{q}^{2}=0.59, \mathrm{r}^{2}=\right.$ 
2780.75 and SDEP = 0.44) (Table S2) was obtained after two cycles of FFD with the

279 pharmacophore-based aligned set of conformations on Tiagabine template.

280 Multiple linear regression analysis using Leave One Out (LOO) cross validation

281 (Elisseeff and Pontil, 2003) of the final model resulted in a plot of observed versus predicted

282 inhibitory potencies $\left(-\log \mathrm{IC}_{50}\right)$ of the training data, as shown in Fig. 3. All the compounds in the

283 training set (filled circles), test set (hollow circles) and validation set (triangles) are well-

284 predicted, with a difference of less than one log unit between the observed and the predicted

285 biological activity values with the exception of compound $\mathbf{3 5}$, for which the predicted activity (-

$286 \log \mathrm{IC}_{50}$ ) value is $1.52 \log$ units greater as compared to experimental activity value (Fig. 3).

287 However, no outlier has been identified in the test set $\left(\mathrm{R}^{2}: 0.53\right.$, Table S3) and the validation set

$288\left(\mathrm{R}^{2}\right.$ : 0.57). Briefly, the higher predicted activity value for compound 35 (actual $-\log$ $289 \mathrm{IC}_{50}$ /predicted $-\log \mathrm{IC}_{50}:-1.44 / 0.08$ ) (outlier in Fig. 3) is may be due to its high lipophilicity $290(\log \mathrm{P}=7.80)$ which may lead to poor bioavailability and thus, overall poor experimental 291 efficacy.

292 Fig. 4 shows PLS coefficients correlograms of the final GRIND model. Positive and 293 negative peaks in auto and cross correlograms of different variables (O, N1, DRY and TIP) 294 elucidate their positive or negative contributions, respectively, towards the inhibitory potency ($\left.295 \log \mathrm{IC}_{50}\right)$ against hGAT1. Additionally, it depicts the 3D structural features and their mutual 296 distances that best describe their roles in the inhibition of hGAT1. It is evident from the PLS co297 efficient correlogram that the O-O, N1-N1, DRY-N1, O-N1 and TIP-TIP variables have major 298 influences on the inhibitory potency of the data set.

299 N1-N1 variables in Fig. 4 depict the presence of two hydrogen bond acceptor groups 300 within the molecules that are present at a mutual distance of 8.00-8.40 $\AA$ in highly active (0.049$3010.75 \mu \mathrm{M})$ antagonists of hGAT1. In the current data set, it represents a distance between the 302 carbonyl and hydroxyl groups of the $\mathrm{COOH}$ attached at the ortho, meta and para positions of the

303

304

305

306

307

308 piperidine ring in the nipecotic acid derivatives, as shown in Fig. 4A. All the compounds in the present data set (class $\mathrm{A}, \mathrm{B}$ and $\mathrm{C}$ ) possess $\mathrm{COOH}$ groups attached at the ortho, meta or para positions of the piperidine ring. Compound $\mathbf{3 7}$ in class $\mathrm{C}$ is the only exception, where the $\mathrm{COOH}$ group has been substituted with an isoxazol ring. The N1-N1 correlogram for compound 37 represents a distance of 8.00-8.40 $\AA$ between the tertiary nitrogen of the piperidine ring and the carbonyl group of the isoxazol ring. Additionally, the N1-N1 pair of variables at a longer 
309 distance range of 14.00-14.40 $\AA$ shows a negative contribution towards the hGAT1 inhibition 310 potential and, thus, has been identified in the least active $(5.00-38 \mu \mathrm{M})$ compounds in the data 311 set. Briefly, it represents a distance between the carbonyl oxygen of the COOH group and the 312 ether group present either in the linker region or in the bulky aromatic substituents, as shown in 313 Fig. 4B.

314 Interestingly, a sharp positive peak in the O-N1 correlogram in Fig. 4 demonstrates that a 315 hydrogen bond acceptor at a distance of 5.60-6.00 $\AA$ from a hydrogen bond donor region has a 316 positive effect on the inhibitory potency against hGAT1. Within the present data set, the 317 hydrogen bond donor region represents the protonated nitrogen of the piperidine ring, while the $318 \mathrm{COOH}$ group provides the hydrogen bond acceptor region, as shown in Fig. 4C. This distance 319 has been identified in the most active $(0.049-0.75 \mu \mathrm{M})$ hGAT1 antagonists and is absent in the 320 least active (5.00-38 $\mu \mathrm{M})$ antagonists. This further strengthens the importance of the carbonyl 321 group and the protonated nitrogen for the high inhibitory potency of hGAT1 antagonists. 322 Additionally, the O-N1 pair of probes at a larger distance range of 10.40-10.80 $\AA$, as shown in 323 Fig. 4D, has been identified in the least active $(6.56-78 \mu \mathrm{M})$ compounds within the data series 324 and, thus, has a negative effect on the overall inhibitory potency against hGAT1. In the present 325 data series, this represents a distance between the protonated nitrogen (hydrogen bond donor) of 326 the piperidine ring and the methoxy substitution of the diaryl moieties (hydrogen bond acceptor), 327 as shown in Fig. 4D. Previously, the importance of the protonated nitrogen group in the 328 piperidine ring of hGAT1 antagonists has been demonstrated by Zheng et al. by CoMFA 329 analysis of N-diarylalkenyl-piperidinecarboxylic acid analogues (Zheng et al., 2006). Thus, it is 330 tempting to speculate that the methoxy groups at the N-diarylalkenyl rings in the data series may have a negative effect on the inhibitory potency against hGAT1.

Similarly, the DRY-N1 pair of probes in Fig. 4 represents one hydrophobic region (DRY) and one hydrogen bond acceptor variable region within the molecules (N1). Both features have 334 been identified at a mutual distance of 10.40-10.80 $\AA$ in highly active compounds (0.049-0.75 $\mu \mathrm{M})$ and at a distance of 6.40-6.80 $\AA$ in the least active compounds (4.34-78 $\mu \mathrm{M})$. In highly active compounds, this represents the distance between aromatic moieties (thiophene rings, benzene rings and tricyclic rings) and the $\mathrm{COOH}$ group at the piperidine, proline, pyrrolidine, or azetidine ring, as shown in Fig. 4E. However, in the least active compounds it represents a 339 distance between the aromatic moieties and the ether group in the linker region, as shown in Fig. 
340 4F. Interestingly, all the selected compounds of classes A, B and C follow the distance pattern of

341 highly active compounds in the DRY-N1 correlogram. Moreover, compound $37\left(\mathrm{IC}_{50}=38 \mu \mathrm{M}\right)$

342 of class $\mathrm{C}$ shows a distance of 6.40-6.80 $\AA$ between the carbonyl group of the isoxazol ring and

343 the benzene ring substituent at the linker region, which may provide a reason for the low

344 biological activity of compound 37.

345 Furthermore, the O-O correlogram in Fig. 4 shows the presence of two hydrogen bond 346 donors at a mutual distance of 6.00-6.40 $\AA$ within the least active (10-78 $\mu \mathrm{M})$ compounds and, 347 thus, may contribute negatively towards the inhibitory potency of the data set against hGAT1.

348 For instance, in compound 32 this distance is associated with the ether group present in the 349 hydrophilic chain and the para-monofluoro groups attached to the aromatic rings, as shown in 350 Fig. 4G. Thus, it may be inferred that attachment of any electronegative atom to the ortho or para 351 positions of the aromatic moieties is not favorable for achieving high biological activity values in 352 this data series. Similarly, another example of the O-O correlogram is presented by compound 36 353 of class $\mathrm{B}$, for which this variable represents the distance between the ether linker region of the 354 hydrophilic chain and the para-methoxy group attached at the $\mathrm{R}_{2}$ group, as shown in Fig. $4 \mathrm{H}$.

355 Furthermore, the TIP-TIP correlogram in Fig. 4 represents two molecular boundaries of 356 methoxy substitutions on the aromatic moieties at a mutual distance of 12.40-12.80 $\AA$ in the least

357 active compounds $(11.00-78 \mu \mathrm{M})$. In the present data set, this represents the distance between the 358 para-methoxy substituents at $R_{1}$ and $R_{2}$ of the aromatic rings in class B (Fig. 4I) and the distance 359 between benzene ring attached at the linker region and the isoxazol ring in compound $\mathbf{3 7}$ of class $360 \mathrm{C}\left(\mathrm{IC}_{50}=38 \mu \mathrm{M}\right)$. Interestingly, the results of the TIP-TIP correlogram are further strengthened 361 by our findings for the O-O correlogram that addition of para-methoxy groups to the aromatic 362 moieties results in reduced biological activity. Interestingly, N1-TIP correlogram in Fig. 4 363 represents a distance of 18.00-18.40 $\AA$ between the molecular boundary depicted by the $\mathrm{R}_{1}$ and $364 \mathrm{R}_{2}$ substitutions of the aromatic rings and the $\mathrm{COOH}$ group attached to the piperidine ring in the 365 least active compounds. Thus, it may suggest the negative impact of $R_{1}$ and $R_{2}$ substitutions 366 towards hGAT1 inhibitory potency as the importance of $\mathrm{COOH}$ group is already evident from 367 N1-N1 and O-N1 variables. Overall, a brief summary of all the specific probes contributing 368 towards the activity of hGAT1 antagonists is presented in Table 1.

\section{External validation of the final GRIND model}


371 published data set of 15 nipecotic acid derivatives possessing an alkyne type spacer (linker 372 region) bridging the polar region at the aromatic rings, as shown in Table 2, was used for 373 external validation (Lutz et al., 2017).

374 Overall, the $\mathrm{IC}_{50}$ values of the data set range between 0.07 and $3.80 \mu \mathrm{M}$ (Table 2). All the 375 biological testing results (inhibitory potencies) were the mean of three independent experiments $376 \pm$ the standard error of the mean (SEM) (Lutz et al., 2017). Remarkably, all the compounds in the 377 external validation set are well-predicted with $\mathrm{R}^{2}$ value of 0.57 (Fig. 3, represented by triangles), 378 and exhibiting a difference of less than one log unit between the experimental and predicted 379 inhibitory values, as shown in Table 2.

\section{Homology modeling of hGAT1}

Based on the alignment (representing 66\% sequence similarity (Fig. S4); equivalent

382

383

384

385

386

387

388

389

390

391

392

393

394

395

396

397

398

399

400

to $46 \%$ sequence identity) hGAT1 models in open to out conformation were constructed using dDAT as a template as explained in methodology section. The Ramachandran plot of the final selected model with best ERRAT value (81.28) in comparison to ERRAT values of rest of the models (range: 69.93-80.00) and Verify3D score (83.24\%) has shown four residues in the outlier region, including F174 (EL2), N176 (EL2), R419 (TM9), and V528 (TM11). In order to further improve the model quality, final selected model was energy minimized via LigX using MMFF94x in MOE v2013.0802 (Halgren, 1996;MOE, 2013.08). After the energy minimization, the model was re-evaluated by PROCHECK and none of the residues were observed in the disallowed region (Fig. S5). The Ramachandran plot displayed 93.2\% of the residues of hGAT1 in most favored regions, 5.9\% residues in additionally allowed regions, $0.9 \%$ residues in generously allowed regions, and $0.0 \%$ residues in disallowed regions. Similarly, the ERRAT (87.92) and Verify3D (85\%) scores were also improved compared to the model before energy minimization. This further shows the reliability of the final model.

Briefly, ERRAT score provides information about the residues that contribute towards the lower percentage/score of the hGAT1 model (i.e., residues 165 to 180). This is may be because EL2 in hGAT1 contains a greater number of residues than in AaLeuT and dDAT. These may be the flanking residues of this loop. Furthermore, Gether et al. reported that binding of the ligand to the transporter in the open-to-out conformation results in the penetration of extracellular loop 4 (EL4) deep inside the hGAT1 binding site (i.e., TM1 residues), leading to 
401 the formation of a lid-type structure that seals the ligand into the hydrophobic core/region of the

402 binding cavity. This confirms that the binding of the substrate to the transporter in its open-to-out 403 form involves a major conformational change in EL4 (Gether et al., 2006). In the constructed 404 hGAT1 model, EL4 penetration into the hydrophobic region of the binding cavity has resulted in 405 the adjustment of bi- or tri-aromatic/ cyclic moieties of hGAT1 antagonists. Overall, the final 406 model has 12 transmembrane (TM) segments, two $\mathrm{Na}^{+}$ions and one $\mathrm{Cl}^{-}$ion along with the 407 bridging extracellular and intracellular loops. One side of the hydrophobic cavity around cyclic 408 moieties is defined by the residues W68 (TM1b), Y139 (TM3), Y140 (TM3) and I143 (TM3) 409 and the other by F294 (TM6a), S359 (EL4a), D451 (TM10), and S456 (TM10). The final 410 structural model of hGAT1 in the open-to-out conformation, along with the two $\mathrm{Na}^{+}$, one $\mathrm{Cl}^{-}$and 411 binding pocket residues are shown in Fig. S6.

\section{Molecular docking of selected ligands in hGAT1}

\section{Structure-activity relationship}

On the basis of the structure activity relationship, 4 compounds each from classes $\mathrm{A}$ and $\mathrm{B}$ and 5 compounds from class $\mathrm{C}$ have been selected for further structure-based studies, as shown in Table 3.

Briefly, class A includes N-diarylalkenyl piperidine $\mathrm{COOH}$ derivatives (Table S1) with a $\mathrm{COOH}$ group substituent at the ortho, meta or para position of the piperidine ring. $\mathrm{COOH}$ group in compounds $\mathbf{2}$ and $\mathbf{6}$ was R-configured however, stereochemistry of rest of the compounds in class $\mathrm{A}$ was not specified and thus, represent racemic mixtures. Compound 2 (Tiagabine, $\mathrm{IC}_{50}=$ $0.049 \mu \mathrm{M}$ ), the only FDA approved antiepileptic drug, was selected for molecular docking to investigate its binding hypothesis and to compare it with rest of the analogues in class A. Compound $6\left(\mathrm{IC}_{50}=0.34 \mu \mathrm{M}\right)$ was selected to evaluate the one order of magnitude decrease in its inhibitory potency compared to compound 2, which may be due to the presence of bulky substituents $\left(-\mathrm{CH}_{2} \mathrm{OC}_{6} \mathrm{H}_{5}\right)$ at $\mathrm{R}_{1}$ and $\mathrm{R}_{2}$ in compound 6 compared to methyl substituents at the same positions in compound 2. Interestingly, compounds 8 and $\mathbf{1 5}$ have the same bulky substituents $\left(-\mathrm{CH}_{2} \mathrm{OC}_{6} \mathrm{H}_{5}\right)$ at $\mathrm{R}_{1}$ and $\mathrm{R}_{2}$ as compound 6; however, an approximate two-fold decrease in the biological activity has been observed, i.e., $6\left(\mathrm{IC}_{50}=0.34 \mu \mathrm{M}\right)>\mathbf{8}\left(\mathrm{IC}_{50}=0.65\right.$ $\mu \mathrm{M})>15\left(\mathrm{IC}_{50}=1.5 \mu \mathrm{M}\right)$ that can be correlated to the position (meta $>$ ortho $>$ para) of the $\mathrm{COOH}$ group on the piperidine ring. Briefly, compound $6\left(\mathrm{IC}_{50}=0.34 \mu \mathrm{M}\right)$, which has the 
432 inhibitory potency against hGAT1 compared to compound $8\left(\mathrm{IC}_{50}=0.65 \mu \mathrm{M}\right)$, which has a

$433 \mathrm{COOH}$ group at the ortho position, and in turn showed two-fold greater activity as compared to

434

435

436

437

438

439

440

441

442

443

444

445

446

447

448

449

450

451

452

453

454

455

456

457

458

459

460

461

462 compound $15\left(\mathrm{IC}_{50}=1.5 \mu \mathrm{M}\right)$, which has a $\mathrm{COOH}$ group at the para position (p-COOH). Therefore, compounds 6, 8 and $\mathbf{1 5}$ were also selected for docking within the binding cavity of hGAT1 to understand the binding hypothesis of the $\mathrm{COOH}$ group at the ortho, meta and para positions of the piperidine ring.

Class B of hGAT1 antagonists consists of ethyl trityl ether derivatives of nipecotic acid that possess a R-configured $\mathrm{COOH}$ group at the meta position $(\mathrm{m}-\mathrm{COOH})$ of the piperidine ring and $R_{1}, R_{2}$ and $R_{3}$ methoxy substituents on the triphenylmethyl group, as shown in Table $S 1$. Compound 14 being the highly active in class $\mathrm{B}\left(\mathrm{IC}_{50}=1.40 \mu \mathrm{M}\right)$, was selected for comparison with the rest of the data. Attachment of a para-methoxy group at $\mathrm{R}_{2}$ in compound 27 results in a 5-fold decrease in the biological activity $(6.9 \mu \mathrm{M})$ compared to compound 14. Interestingly, para-methoxy substituents at $\mathrm{R}_{1}, \mathrm{R}_{2}$ and $\mathrm{R}_{3}$ in compound $36\left(\mathrm{IC}_{50}=30 \mu \mathrm{M}\right)$ and at $\mathrm{R}_{1}$ and $\mathrm{R}_{2}$ in compound $40\left(\mathrm{IC}_{50}=43 \mu \mathrm{M}\right)$ result in a decrease of approximately two orders of magnitude in the inhibitory potency against hGAT1 compared to compound 14. This suggests that the decrease in the biological activity of compounds $\mathbf{2 7 , 3 6}$ and $\mathbf{4 0}$ compared to compound $\mathbf{1 4}$ is may be due to the presence of para-methoxy groups at $R_{1}, R_{2}$ and $R_{3}$, which might cause steric hindrance within the binding pocket or limit the access of the compounds to the hGAT1 binding pocket.

This is further validated by our findings from the GRIND model that para-methoxy substituents at aromatic moieties and the ether linker group at a mutual distance of 6.00-6.40 (O-O probes) have a negative effect on the hGAT1 inhibitory potency. Therefore, compounds 27, 36 and 40 were also selected in addition to compound $\mathbf{1 4}$ for further molecular docking studies to probe the effect of para-methoxy substitutions on aromatic moieties on binding within the active site of hGAT1.

Compounds 1, 3, 4, 37 and 84, which are diaryl derivatives of nipecotic acid (all having racemic $\mathrm{COOH}$ groups with the exception of compound $\mathbf{3 7}$ as shown in Table S1) were selected from class $\mathbf{C}$ to elucidate the effect of the attachment of diaryl and the piperizine derivatives at the linker region on the biological activity of hGAT1 antagonists. Compound 1 (NNC-711), the 2-benzhydrylideneamino derivative of nipecotic acid, was selected as a reference ligand in the class since it is a known selective inhibitor of hGAT1 $\left(\mathrm{IC}_{50}=0.040 \mu \mathrm{M}\right)($ Kragler et al., 2005). However, replacement of the $-\mathrm{C}=\mathrm{NO}$ - in compound $\mathbf{1}$ with $-\mathrm{C}=\mathrm{CH}-$ and $-\mathrm{CHO}$ - in compounds 3 
463 (SK\&F-100330A) and 4 (CI-966) resulted in one order of magnitude decrease in the biological 464 activities of compounds $3\left(\mathrm{IC}_{50}=0.20 \mu \mathrm{M}\right)$ and $4\left(\mathrm{IC}_{50}=0.26 \mu \mathrm{M}\right)$ compared to $1\left(\mathrm{IC}_{50}=0.040\right.$ $465 \mu \mathrm{M})$. This decrease in biological activity has been correlated to an increase in the $\log \mathrm{P}$ values, $466 \log \mathrm{P} 4(5.45)>\log \mathrm{P} 3(4.19)>\log \mathrm{P} 1$ (3.79), which may reflect the importance of polar groups 467 in interactions within the binding pocket of hGAT1. As a result, compounds $\mathbf{1}, \mathbf{3}$ and $\mathbf{4}$ were 468 selected for further molecular docking and ligand-protein interaction profiling. Additionally, 469 compound 84 was selected to compare its ligand-protein interaction profile with compound 3 . In 470 compound 37, replacement of the $\mathrm{COOH}$ group with 4,5,6,7-tetrahydroisoxazolo[4,5-c]pyridin4713 -ol resulted in a three order of magnitude decrease in the inhibitory potency of the compound $472\left(\mathrm{IC}_{50}=38 \mu \mathrm{M}\right)$ compared to compound $1\left(\mathrm{IC}_{50}=0.040 \mu \mathrm{M}\right)$. Therefore, compound 37 was 473 selected to elucidate the effect of nipecotic acid on the inhibitory potency against hGAT1. This 474 may point towards the importance of nipecotic acid in obtaining high inhibitory potency against 475 hGAT1.

476 Briefly, all the selected compounds from class A $(\mathbf{2}, \mathbf{6}, \mathbf{8}, \mathbf{1 5})$, class B $(\mathbf{1 4}, \mathbf{2 7}, \mathbf{3 6}, \mathbf{4 0})$ and 477 class $C(\mathbf{1}, \mathbf{3}, \mathbf{4}, \mathbf{3 7}, \mathbf{8 4})$ were docked into the binding site of the hGAT1 model as explained in 478 the methods section. To remove any biases in the docking protocol, 100 poses per ligand were 479 generated using the GOLD score fitness function. However, the fitness function in Gold score is 480 481 482 optimized for the prediction of binding positions rather than binding affinities (Jones et al., 1995) therefore, a poor correlation $\left(\mathrm{R}^{2}: 0.02\right)$ has been observed between biological activity $\left(-\log \mathrm{IC}_{50}\right)$ and top scored pose of each ligand (Fig. S7, Table S4). Therefore, in order to remove any biases in the pose selection criteria, for the final ligand-protein interaction analysis, only one cluster exhibiting maximum docked ligands from each class was selected on the basis of the SAR and 485 mutagenesis data, followed by energy minimization of the final ligand-protein complexes (Fig. 486 S7, Table S4) (Halgren, 1996;MOE, 2013.08).

Briefly, a total number of 14 clusters of docking solutions of compounds in class A, 12 clusters of binding conformations of compounds in class B, and 4 clusters of binding solutions 489 for the compounds in class $\mathrm{C}$ have been identified that contained the maximum number of 490 docked ligands. Briefly, all 14 clusters of class A contained $3(\mathbf{2}, \mathbf{8}, \mathbf{1 5})$ out of 4 docked ligands, 491 12 clusters of class B also contained $3(\mathbf{1 4}, \mathbf{2 7}, \mathbf{3 6})$ out of 4 docked ligands and 4 clusters of class 492 C contained $4(\mathbf{1}, \mathbf{3}, \mathbf{3 7}, \mathbf{8 4})$ out of 5 docked ligands as shown in Fig. 5. 
The binding position of all the 14 clusters in class A was approximately the same.

494 However, the binding conformations of the docking solutions in each cluster were different.

495 Therefore, the interaction patterns of all 14 clusters of class A were explored and only one

496 cluster that is strengthened by the SAR and mutagenesis data was selected for the final ligand-

497 protein interaction analysis. A similar selection procedure was repeated for the 12 clusters of

498 binding solutions for the selected compounds in class B and the 4 clusters of binding poses for

499 the selected compounds in class C. Sodium ion (represented by Na1) was involved in

500 interactions with the $\mathrm{COOH}$ attached to the piperidine ring in all three classes, as well as with the 501 amino acid residues A61, N66, S295, and N327, as shown in Fig. 6A.

502 Overall, the winning cluster of class A showed interactions with TM segments 1a, 1b, 6a,

$5036 \mathrm{~b}$ and 10 (Fig. 6B). It has been observed that the carbonyl group at the meta position of the 504 piperidine ring in compound 2 shows a strong hydrogen bonding interaction with the - NH group 505 of G65, whereas the thiophene rings of the common scaffold shows a $\pi-\pi$ interaction with Y140 506 at TM3 and W68 at TM1b (Fig. 7A). Additionally, F294 of TM6a shows a strong hydrogen 507 bonding interaction with the protonated nitrogen of the piperidine ring. In compounds 8 and 15 508 hydrogen bonding is observed between the - NH group of $\mathrm{G} 65$ and the $\mathrm{OH}$ group of the $\mathrm{COOH}$, 509 as shown in Fig. 7B. Thus, the one order of magnitude decrease in the inhibitory potency of 510 compounds 8 and 15 compared to compound $\mathbf{2}$ may be attributed to a decrease in the hydrogen 511 bonding strength due to a shift in the position of the hydrogen bond from the carbonyl group (in 512 compound 2) to the hydroxyl group (in compounds 8 and 15). The $\mathrm{COOH}$ flipping is might be 513 due to the shift of the $\mathrm{COOH}$ group from the meta position to the ortho and para positions of the 514 piperidine ring in compounds 8 and 15, respectively.

515 Additionally, the ligand-protein interaction pattern of the final docking poses of 516 compounds 8 and $\mathbf{1 5}$ reveals that bulky substituents at the $\mathrm{R}_{1}$ and $\mathrm{R}_{2}$ positions are more exposed 517 to the extracellular environment (Fig. 8). This disrupts the $\pi-\pi$ stacking between the phenyl ring 518 of Y140 and the thiophene ring associated with $R_{1}$ in compounds 8 and 15, which was present in 519 compound 2 . However, the $\pi-\pi$ interaction between the thiophene ring of the common scaffold of 520 class A and the indole ring of W68 at TM1b and the hydrogen bonding interaction between the 521 protonated nitrogen of the piperidine ring and F294 at TM6a remained intact, as shown in Fig.

522 7B. Therefore, this shows that the bulky substituents on the thiophene rings in compounds 8 and 52315 result in a loss of the interaction with Y140, which is known to be critical for the transport 
524 activity of GATs (Bismuth et al., 1997). These results agree with our SAR data showing that the

525 attachment of bulky groups at the $R_{1}$ and $R_{2}$ positions in class $A$, together with the positioning of 526 the $\mathrm{COOH}$ on the piperidine ring, may affect the inhibitory potency against hGAT1. Overall, the 527 amino acid residues G65, W68, Y140 and F294 are involved in interactions with the N528 diarylalkenyl piperidine $\mathrm{COOH}$ derivatives within the hGAT1 binding cavity.

529 Similarly, out of 12 clusters of binding solutions of compounds in class B, only one 530 cluster (Fig. 6B), which contains the binding poses of compounds 14, 27 and 36, followed the 531 SAR pattern in their ligand-protein interaction profile. However, the rest of the clusters shared 532 similar overall binding positions within the binding pocket of hGAT1. As all the compounds in 533 class $\mathrm{B}$ have a meta- $\mathrm{COOH}$ and a protonated nitrogen in piperidine ring, their binding solutions 534 showed a very similar pattern of hydrogen bonding interactions with the residues G65 and F294 535 and with $\mathrm{Na} 1$, as shown by compound 2 in class A. However, the benzene ring of the common 536 scaffold in compound $\mathbf{1 4}$ shows a $\pi-\pi$ interaction with the indole ring of W68 at TM1b (Fig. 7C). 537 Additionally, both compounds 27 and 36 (Fig. 7D) shows hydrogen bonding interactions 538 between the ether group in the linker region and the pyrrole ring of W68. Similar to the 539 compounds 8 and 15 in class A, the methoxy substitutions on the aromatic moieties in 540 compounds 27 and 36 projected out of the hGAT1 binding pocket (Fig. 8). They may require a 541 large space in the cavity compared to compound 14, and as a result showed no interaction with 542 the residues in the hGAT1 binding pocket.

543 Similar to the selected clusters of class A and B, the final cluster of class C followed the 544 SAR and mutagenesis data. The final binding solutions for class $C$ contain compounds 1, 3, 37 545 and 84, which are located in the vicinity of TM1a, TM1b, TM4, TM6a and TM6b (Fig. 6B). The 546 selected compounds in class $\mathrm{C}$ show a $\pi-\pi$ interaction with W68, followed by a similar hydrogen 547 bonding interaction pattern at the protonated nitrogen atom ( $\mathrm{NH}---\mathrm{O}=\mathrm{C}-\mathrm{F} 294)$ and at the $\mathrm{COOH}$ 548 group (C=O---HN-G65) (Fig. 7E, 7F) (ligplot shown in Fig. S8) as that discussed for classes A 549 (compound 2) and B. However, compound 37 is an exception in which the $\mathrm{COOH}$ is replaced 550 with a five membered isoxazol ring. The residues surrounding Nal are similar in the case of 551 compound 37, as shown in Fig. 7G. Thus, the two to three orders of magnitude decrease in the 552 inhibitory potency of compound $\mathbf{3 7}$ compared to its congeners is might be due to lack of $\mathrm{COOH}$ 553 group and its associated interactions within the hGAT1 pocket.

\section{Discussion}


In present study, we demonstrated the importance of $\mathrm{COOH}$ group towards hGAT1

556 inhibition. Our final GRIND model mapped the distances of important pharmacophoric features

557 of the ligands, including one hydrogen bond acceptor (N1), one hydrogen bond donor (O) and

558 one hydrophobic (Dry) probe from the $\mathrm{COOH}$ group at meta- position of the piperidine ring as

559 shown in Fig. 9 which reflect that $\mathrm{COOH}$ group at meta position of the piperidine ring may

560 provide an important interaction point within the binding cavity of hGAT1.

$561 \quad$ Fig. 9 is further validated by our SAR and docking protocol, where all three classes A, B

562 and $\mathrm{C}$ showed interactions between the protonated nitrogen atom and F294 and between the

$563 \mathrm{COOH}$ group and G65. The effect of the $\mathrm{COOH}$ group, as well as the protonated nitrogen atom,

564 on the inhibitory potency of hGAT1 modulators has been previously demonstrated by various

565 authors (Zhao et al., 2005;Fülep et al., 2006;Kragler et al., 2008;Pizzi et al., 2011). Therefore,

566 three orders of magnitude decrease in inhibitory potency of compound 37 as compared to

567 compound $\mathbf{1}$ is may be due to absence of $\mathrm{COOH}$ group in its chemical scaffold.

Furthermore, $\mathrm{COOH}$ groups at the meta position of docked antagonists (class $\mathrm{A}$; compound 2 and class B) has been observed in the equatorial conformation with respect to Na1 in the hGAT1 model (Fig. 6A). Thus, it suggests that the meta positioning of the equatorial $\mathrm{COOH}$ group is more favorable for obtaining more potent hGAT1 antagonists compared to

572 equatorial ortho or para $\mathrm{COOH}$ positioning. This observation agrees with previous studies by 573 Skovstrup et al. and Jurik et al., who demonstrated that the R-configured COOH group in an 574 axial conformation with respect to $\mathrm{Na} 1$ is involved in intramolecular hydrogen bonding with the 575 protonated nitrogen of the piperidine ring (Skovstrup et al., 2010; Jurik et al., 2015).Thus, 576 attachment of bulky groups (i.e., $\mathrm{COOH}$ ) in an axial configuration with respect to Na1 is not 577 favorable (Jurik et al., 2015). Previously, various authors proposed R-configured COOH group in 578 hGAT1 antagonists as favorable binding conformation (Borden et al., 1994; Wermuth, 579 2008;Schmidt et al., 2017). However, in present study the stereochemical effect of hGAT1 580 antagonists in ligand protein interaction could not be considered due to lack of complete 581 stereoisomers data.

582 Our results also demonstrate the negative impact of methoxy substitutions at $R_{1}, R_{2}$ or $R_{3}$ 583 positions of di- or tri-aryl rings and the ether linker group towards GAT1 inhibition. We 584 hypothesize that 7-fold decrease in the biological activity of $27\left(\mathrm{IC}_{50}=6.9 \mu \mathrm{M}\right)$ and one order of 585 magnitude decrease in the biological activity of $\mathbf{3 6}\left(\mathrm{IC}_{50}=30 \mu \mathrm{M}\right)$ as compared to compound 14 
$586\left(\mathrm{IC}_{50}=1.4 \mu \mathrm{M}\right)$ is may be due to attachment of methoxy groups at $\mathrm{R}_{1}, \mathrm{R}_{2}$ or $\mathrm{R}_{3}$. This is supported 587 by the O-O (Fig. 4G \& 4H) and TIP-TIP correlograms (Fig. 4I) of the final GRIND model. Both 588 distance features (O-O and TIP-TIP) are arising from methoxy or ether linker substituents and 589 show a negative effect on the inhibitory potency against hGAT1. In compounds $\mathbf{2 7 ,} \mathbf{3 6}$ and $\mathbf{4 0}$ of 590 class B, these map the distance between the ether in the linker region and the para-methoxy 591 groups at the $\mathrm{R}_{2}$ position. Hence the biological activity values of $\mathbf{2 7 ,} 36$ and $\mathbf{4 0}(6.9 \mu \mathrm{M}, 30 \mu \mathrm{M}$ 592 and $43 \mu \mathrm{M})$ are significantly reduced compared to compound $14(1.4 \mu \mathrm{M})$, which lacks 593 electronegative atom substituents at the $\mathrm{R}_{1}, \mathrm{R}_{2}$ and $\mathrm{R}_{3}$ positions. Additionally, two orders of 594 magnitude decrease in the inhibitory potency of compounds in class B compared to compounds 595 in class A is may be attributed to the presence of the para-methoxy group in the common 596 scaffold for class B compared to common scaffold of class A. This is further strengthened by a 597 study by Pizzi et al., who studied the effect of ortho, meta and para substitutions on 4,4598 diphenylbut-3-enyl derivatives and observed that biological activity against hGAT1 reduces with 599 the substitution of methyl, chloride, fluoride and bromide at the ortho position of the di-aryl rings 600 (Pizzi et al., 2011). This is also strengthened by the ligand protein interaction analysis of the final 601 docking poses of compounds of class A, B and C which elucidate that the bulky substitutions at $602 \mathrm{R}_{1}$ and $\mathrm{R}_{2}$ positions of di- or tri-aryl rings are projected out of the binding cavity, exposed to the 603 extracellular environment and thus, are not well fitted within the binding cavity of hGAT1.

Overall, the ligand-protein interactions profile of selected hGAT1 inhibitors of calss A, B 605 and C showed significant role of G65, W68, Y140 and F294 amino acid residues within the 606 hGAT1 binding pocket. Previously, Baglo et al., revealed hydrogen bonding and $\pi-\pi$ stacking 607 with G65, N66, Y140, F294 and S295by docking substrate-like small molecules (i.e., 5608 aminolevulinic acid (ALA), and methyl ester of ALA (MAL)) in the hGAT1 model (Baglo et al., 609 2013). Additionally, various structure-based studies identified the binding hypothesis of 610 Tiagabine and demonstrated the role of the amino acid residues W68, Y139, Y140, I143, F294, 611 A358 and S359 in the formation of hydrophobic pockets in hGAT1 (Skovstrup et al., 2010;Jurik 612 et al., 2015). Overall, the ligand-protein interaction profiles of the final binding solutions of 613 hGAT1 inhibitors from classes $\mathrm{A}, \mathrm{B}$ and $\mathrm{C}$ were compared to the already known interaction 614 patterns of other classes of respective modulators, as shown in Fig. S9. It is evident from Fig. S9 615 that the N-diarylalkenyl piperidine derivatives of class A show an overlap with all of the residues 616 already reported in the literature except D451. Similarly, the interaction profile of class B agrees 
617 with that of class A, with the exception of S456. The nipecotic acid derivatives in class C 618 completely agree with the literature, thus further validating the binding hypothesis of selected 619 hGAT1 antagonists.

620 On the basis of these findings, further analyses will focus on virtual screening followed 621 by activity and ADME profiling of subsequent hits and optimization of respective chemical 622 scaffolds structures to identify new arsenal of hGAT1 antagonists with improved efficacy and 623 better ADME properties.

\section{Conclusion}

The current study probes the 3D structural features and binding hypothesis of hGAT1 626 inhibitors in the binding pocket of an in-house homology model of hGAT1 in the open-to-out conformation. Overall, our GRIND model illustrated the importance of two hydrogen bond acceptor groups at mutual distance of 8.00-8.40 $\AA$, one hydrogen bond donor and one hydrophobic group at distance of 5.60-6.00 $\AA$ and10.40-10.80 $\AA$, respectively from one of the hydrogen bond acceptors for achieving high biological activity against hGAT1. However, the docking studies of nipecotic acid and N-diarylalkenyl piperidine analogs in the binding pocket of the hGAT1 model emphasized that the protonated nitrogen atom is oriented towards the extracellular side of the binding pocket due to the attachment of large hydrophobic moieties. In addition, Nal and the residues G65, W68, Y140 and F294 in the binding pocket showed dominant interactions with the $\mathrm{COOH}$ group, aromatic moieties and the protonated nitrogen atom in the hGAT1 antagonists, respectively, that are important for achieving high activity against hGAT1. Both the GRIND model and the docking studies revealed that a meta-COOH group attached to the piperidine ring of hGAT1 antagonists is more favorable for interactions compared to an ortho or para substituted $\mathrm{COOH}$ group. Moreover, the hydrogen bonding and the specific shape/orientation of the antagonists were found to be significant for achieving highly potent hGAT1 antagonists. Overall, we anticipate that the current study may assist the neurological disorders.

\section{4 \\ References}


645 Alexander, D., Tropsha, A., and Winkler, D.A. (2015). Beware of R 2: simple, unambiguous

646

647

648

649

650

651

652

653

654

655

656

657

658

659

660

661

662

663

664

665

666

667

668

669

670

671

672

673

674

675 assessment of the prediction accuracy of QSAR and QSPR models. Journal of chemical information and modeling 55, 1316-1322.

Alexander, S., Mathie, A., and Peters, J. (2007). Cell-Surface Transmitter Transporters. British Journal of Pharmacology 150, S135-S142.

Alexander, S.P., Mathie, A., and Peters, J.A. (2011). Guide to receptors and channels (GRAC). British journal of pharmacology 164, S1-S2.

Andersen, K.E., Sørensen, J.L., Lau, J., Lundt, B.F., Petersen, H., Huusfeldt, P.O., Suzdak, P.D., and Swedberg, M.D. (2001). Synthesis of Novel $\gamma$-Aminobutyric Acid (GABA) Uptake Inhibitors. 5. 1 Preparation and Structure-Activity Studies of Tricyclic Analogues of Known GABA Uptake Inhibitors. Journal of medicinal chemistry 44, 2152-2163.

Anderson, C.M., Kidd, P.D., and Eskandari, S. (2010). GATMD: $\gamma$-aminobutyric acid transporter mutagenesis database. Database 2010, baq028.

Baglo, Y., Gabrielsen, M., Sylte, I., and Gederaas, O.A. (2013). Homology Modeling of Human $\gamma$-Butyric Acid Transporters and the Binding of Pro-Drugs 5-Aminolevulinic Acid and Methyl Aminolevulinic Acid Used in Photodynamic Therapy.

Baroni, M., Costantino, G., Cruciani, G., Riganelli, D., Valigi, R., and Clementi, S. (1993). Generating Optimal Linear PLS Estimations (GOLPE): An Advanced Chemometric Tool for Handling 3D-QSAR Problems. Quantitative Structure-Activity Relationships 12, 920.

Bicho, A., and Grewer, C. (2005). Rapid substrate-induced charge movements of the GABA transporter GAT1. Biophysical journal 89, 211-231.

Bismuth, Y., Kavanaugh, M.P., and Kanner, B.I. (1997). Tyrosine 140 of the $\gamma$-aminobutyric acid transporter GAT-1 plays a critical role in neurotransmitter recognition. Journal of Biological Chemistry 272, 16096-16102.

Borden, L.A., Dhar, T.M., Smith, K.E., Weinshank, R.L., Branchek, T.A., and Gluchowski, C. (1994). Tiagabine, SK\&F 89976-A, CI-966, and NNC-711 are selective for the cloned GABA transporter GAT-1. European Journal of Pharmacology: Molecular Pharmacology 269, 219-224.

Bouanich, J.-P. (1992). Site-site Lennard-Jones potential parameters for N2, O2, H2, CO and CO2. Journal of Quantitative Spectroscopy and Radiative Transfer 47, 243-250. 
676 Bowie, J.U., Luthy, R., and Eisenberg, D. (1991). A method to identify protein sequences that

677

678

679

680

681

682

683

684

685

686

687

688

689

690

691

692

693

694

695

696

697

698

699

700

701

702

703

704

705

706 fold into a known three-dimensional structure. Science 253, 164-170.

Calapai, G., Crupi, A., Firenzuoli, F., Inferrera, G., Squadrito, F., Parisi, A., De Sarro, G., and Caputi, A. (2001). Serotonin, norepinephrine and dopamine involvement in the antidepressant action of hypericum perforatum. Pharmacopsychiatry 34, 45-49.

Carvill, G.L., Mcmahon, J.M., Schneider, A., Zemel, M., Myers, C.T., Saykally, J., Nguyen, J., Robbiano, A., Zara, F., and Specchio, N. (2015). Mutations in the GABA transporter SLC6A1 cause epilepsy with myoclonic-atonic seizures. The American Journal of Human Genetics 96, 808-815.

Clausen, R.P., Madsen, K., Larsson, O.M., Frølund, B., Krogsgaard-Larsen, P., and Schousboe, A. (2006). Structure-Activity Relationship and Pharmacology of $\gamma$-Aminobutyric Acid (GABA) Transport Inhibitors. Advances in pharmacology 54, 265-284.

Clausen, R.P., Moltzen, E.K., Perregaard, J., Lenz, S.M., Sanchez, C., Falch, E., Frølund, B., Bolvig, T., Sarup, A., and Larsson, O.M. (2005). Selective inhibitors of GABA uptake: synthesis and molecular pharmacology of 4-N-methylamino-4, 5, 6, 7-tetrahydrobenzo [d] isoxazol-3-ol analogues. Bioorganic \& medicinal chemistry 13, 895-908.

Colovos, C., and Yeates, T.O. (1993). Verification of protein structures: patterns of nonbonded atomic interactions. Protein science 2, 1511-1519.

Conti, F., Melone, M., De Biasi, S., Minelli, A., Brecha, N.C., and Ducati, A. (1998). Neuronal and glial localization of GAT-1, a high-affinity $\gamma$-aminobutyric acid plasma membrane transporter, in human cerebral cortex: With a note on its distribution in monkey cortex. Journal of Comparative Neurology 396, 51-63.

Conti, F., Zuccarello, L.V., Barbaresi, P., Minelli, A., Brecha, N.C., and Melone, M. (1999). Neuronal, glial, and epithelial localization of $\gamma$-aminobutyric acid transporter 2, a high-affinity $\gamma$-aminobutyric acid plasma membrane transporter, in the cerebral cortex and neighboring structures. Journal of Comparative Neurology 409, 482-494.

Dalby, N.O. (2000). GABA-level increasing and anticonvulsant effects of three different GABA uptake inhibitors. Neuropharmacology 39, 2399-2407.

Dhar, T.M., Borden, L.A., Tyagarajan, S., Smith, K.E., Branchek, T.A., Weinshank, R.L., and Gluchowski, C. (1994). Design, synthesis and evaluation of substituted triarylnipecotic acid derivatives as GABA uptake inhibitors: identification of a ligand with moderate 
707

708

709

710

711

712

713

714

715

716

717

718

719

720

721

722

723

724

725

726

727

728

729

730

731

732

733

734

735

736

affinity and selectivity for the cloned human GABA transporter GAT-3. Journal of medicinal chemistry 37, 2334-2342.

Durán, Á., Martínez, G.C., and Pastor, M. (2008). Development and validation of AMANDA, a new algorithm for selecting highly relevant regions in molecular interaction fields. Journal of chemical information and modeling 48, 1813-1823.

Durán, Á., and Pastor, M. (2011). An advanced tool for computing and handling GRidINdependent. Descriptors. User Manual Version 1.

Elisseeff, A., and Pontil, M. (2003). Leave-one-out error and stability of learning algorithms with applications. NATO science series sub series iii computer and systems sciences 190,111130.

Faust, M.R., Höfner, G., Pabel, J., and Wanner, K.T. (2010). Azetidine derivatives as novel $\gamma$ aminobutyric acid uptake inhibitors: Synthesis, biological evaluation, and structureactivity relationship. European journal of medicinal chemistry 45, 2453-2466.

Fülep, G.H., Hoesl, C.E., Höfner, G., and Wanner, K.T. (2006). New highly potent GABA uptake inhibitors selective for GAT-1 and GAT-3 derived from (R)-and (S)-proline and homologous pyrrolidine-2-alkanoic acids. European journal of medicinal chemistry 41, 809-824.

Gether, U., Andersen, P.H., Larsson, O.M., and Schousboe, A. (2006). Neurotransmitter transporters: molecular function of important drug targets. Trends in pharmacological sciences 27, 375-383.

Gillet, V.J. (2011). Diversity selection algorithms. Wiley Interdisciplinary Reviews: Computational Molecular Science 1, 580-589.

Halgren, T.A. (1996). Merck molecular force field. I. Basis, form, scope, parameterization, and performance of MMFF94. Journal of computational chemistry 17, 490-519.

Hauke, T.J., Wein, T., Höfner, G., and Wanner, K.T. (2018). Novel allosteric ligands of $\gamma$ aminobutyric acid transporter 1 (GAT1) by MS based screening of pseudostatic hydrazone libraries. Journal of medicinal chemistry.

Hellenbrand, T., Höfner, G., Wein, T., and Wanner, K.T. (2016). Synthesis of 4-substituted nipecotic acid derivatives and their evaluation as potential GABA uptake inhibitors. Bioorganic \& medicinal chemistry 24, 2072-2096. 
737 Heng, J.I.T., Moonen, G., and Nguyen, L. (2007). REVIEW ARTICLE: Neurotransmitters

738

739

740

741

742

743

744

745

746

747

748

749

750

751

752

753

754

755

756

757

758

759

760

761

762

763

764

765

766

767 regulate cell migration in the telencephalon. European Journal of Neuroscience 26, 537546.

Hirayama, B.A., Díez-Sampedro, A., and Wright, E.M. (2001). Common mechanisms of inhibition for the $\mathrm{Na}+$ /glucose (hSGLT1) and $\mathrm{Na}+/ \mathrm{Cl}$-/GABA (hGAT1) cotransporters. British journal of pharmacology 134, 484-495.

Ho, B.K., and Brasseur, R. (2005). The Ramachandran plots of glycine and pre-proline. $B M C$ structural biology 5, 14.

Jin, X.-T., Galvan, A., Wichmann, T., and Smith, Y. (2011). Localization and function of GABA transporters GAT-1 and GAT-3 in the basal ganglia. Front. Syst. Neurosci 5.

Jones, G., Willett, P., and Glen, R.C. (1995). Molecular recognition of receptor sites using a genetic algorithm with a description of desolvation. Journal of Molecular Biology 245, 43-53.

Jurik, A., Reicherstorfer, R., Zdrazil, B., and Ecker, G.F. (2013). Classification of High-Activity Tiagabine Analogs by Binary QSAR Modeling. Molecular informatics 32, 415-419.

Jurik, A., Zdrazil, B., Holy, M., Stockner, T., Sitte, H.H., and Ecker, G.F. (2015). A binding mode hypothesis of tiagabine confirms liothyronine effect on $\gamma$-aminobutyric acid transporter 1 (GAT1). Journal of medicinal chemistry 58, 2149-2158.

Kragler, A., Höfner, G., and Wanner, K.T. (2005). Novel parent structures for inhibitors of the murine GABA transporters mGAT3 and mGAT4. European journal of pharmacology $519,43-47$.

Kragler, A., Höfner, G., and Wanner, K.T. (2008). Synthesis and biological evaluation of aminomethylphenol derivatives as inhibitors of the murine GABA transporters mGAT1mGAT4. European journal of medicinal chemistry 43, 2404-2411.

Labute, P. (2008). The generalized Born/volume integral implicit solvent model: estimation of the free energy of hydration using London dispersion instead of atomic surface area. Journal of computational chemistry 29, 1693-1698.

Lennard-Jones, J.E. (1931). Cohesion. Proceedings of the Physical Society 43, 461.

Lovell Sc, D.I., Arendall Wb 3rd, De Bakker Pi, Word Jm, Prisant Mg, Richardson Js, Richardson Dc. (2003). Structure validation by Calpha geometry: phi,psi and Cbeta deviation. Proteins 50, 437-450. 
768 Lüthy, R., Bowie, J.U., and Eisenberg, D. (1992). Assessment of protein models with three769 dimensional profiles. Nature 356, 83.

770 Lutz, T., Wein, T., Höfner, G., Pabel, J., Eder, M., Dine, J., and Wanner, K.T. (2018).

771 Development of new photoswitchable azobenzene based $\gamma$-aminobutyric acid (GABA) 772 uptake inhibitors with distinctly enhanced potency upon photoactivation. Journal of 773 Medicinal Chemistry.

774 Lutz, T., Wein, T., Höfner, G., and Wanner, K.T. (2017). Development of Highly Potent GAT1 775 Inhibitors: Synthesis of Nipecotic Acid Derivatives with N-Arylalkynyl Substituents. 776 ChemMedChem.

777 Madsen, K.K., Ebert, B., Clausen, R.P., Krogsgaard-Larsen, P., Schousboe, A., and White, H.S. 778 (2011). Selective GABA transporter inhibitors tiagabine and EF1502 exhibit mechanistic 779 differences in their ability to modulate the ataxia and anticonvulsant action of the extrasynaptic GABAA receptor agonist gaboxadol. Journal of Pharmacology and Experimental Therapeutics 338, 214-219.

Magrane, M. (2011). UniProt Knowledgebase: a hub of integrated protein data. Database 2011.

Martin, Y.C., Bures, M.G., Danaher, E.A., Delazzer, J., Lico, I., and Pavlik, P.A. (1993). A fast new approach to pharmacophore mapping and its application to dopaminergic and benzodiazepine agonists. Journal of computer-aided molecular design 7, 83-102.

Melone, M., Ciappelloni, S., and Conti, F. (2015). A quantitative analysis of cellular and synaptic localization of GAT-1 and GAT-3 in rat neocortex. Brain Structure and Function 220, 885-897.

Minelli, A., Brecha, N., Karschin, C., Debiasi, S., and Conti, F. (1995). GAT-1, a high-affinity GABA plasma membrane transporter, is localized to neurons and astroglia in the cerebral cortex. Journal of Neuroscience 15, 7734-7746.

Minelli, A., Debiasi, S., Brecha, N.C., Zuccarello, L.V., and Conti, F. (1996). GAT-3, a highaffinity GABA plasma membrane transporter, is localized to astrocytic processes, and it is not confined to the vicinity of GABAergic synapses in the cerebral cortex. Journal of Neuroscience 16, 6255-6264.

Minh, B.Q., Klaere, S., and Von Haeseler, A. (2009). Taxon selection under split diversity. Systematic biology 58, 586-594. 
798 Moe (2013.08). "Molecular Operating Environment, Chemical Computing Group Inc.". 1010

799

800

801

802

803

804

805

806

807

808

809

810

811

812

813

814

815

816

817

818

819

820

821

822

823

824

825

826

827

828

Sherbooke St. West, Suite \# 910, Montreal, QC, Canada, H3A 2R7, 2015.49).

Nakada, K., Yoshikawa, M., Ide, S., Suemasa, A., Kawamura, S., Kobayashi, T., Masuda, E., Ito, Y., Hayakawa, W., and Katayama, T. (2013). Cyclopropane-based conformational restriction of GABA by a stereochemical diversity-oriented strategy: Identification of an efficient lead for potent inhibitors of GABA transports. Bioorganic \& medicinal chemistry 21, 4938-4950.

Nowaczyk, A., Fijałkowski, Ł., Kowalska, M., Podkowa, A., and Sałat, K. (2018). Studies on the Activity of Selected Highly Lipophilic Compounds toward hGAT1 Inhibition. Part II. ACS chemical neuroscience.

Parpura, V., and Haydon, P.G. (2008). Astrocytes in (patho) physiology of the nervous system. Springer.

Petrera, M., Wein, T., Allmendinger, L., Sindelar, M., Pabel, J., Höfner, G., and Wanner, K.T. (2015). Development of Highly Potent GAT1 Inhibitors: Synthesis of Nipecotic Acid Derivatives by Suzuki-Miyaura Cross-Coupling Reactions. ChemMedChem.

Pirttimaki, T., Parri, H.R., and Crunelli, V. (2013). Astrocytic GABA transporter GAT-1 dysfunction in experimental absence seizures. The Journal of physiology 591, 823-833.

Pizzi, D.A., Leslie, C.P., Di Fabio, R., Seri, C., Bernasconi, G., Squaglia, M., Carnevale, G., Falchi, A., Greco, E., and Mangiarini, L. (2011). Stereospecific synthesis and structureactivity relationships of unsymmetrical 4, 4-diphenylbut-3-enyl derivatives of nipecotic acid as GAT-1 inhibitors. Bioorganic \& medicinal chemistry letters 21, 602-605.

Quandt, G., Höfner, G., and Wanner, K.T. (2013). Synthesis and evaluation of N-substituted nipecotic acid derivatives with an unsymmetrical bis-aromatic residue attached to a vinyl ether spacer as potential GABA uptake inhibitors. Bioorganic \& Medicinal Chemistry 21, 3363-3378.

Reith, M.E. (2007). Handbook of Neurochemistry and Molecular Neurobiology: Neural Membranes and Transport. Springer Science \& Business Media.

Release, S. (2017). Schrödinger 2017-1, LLC. New York, NY.

Rud, E., Gederaas, O., Høgset, A., and Berg, K. (2000). 5-aminolevulinic acid, but not 5aminolevulinic acid esters, is transported into adenocarcinoma cells by system BETA transporters. Photochemistry and photobiology 71, 640-647. 
829 Sadowski, J. (2003). 3D structure generation. Handbook of Chemoinformatics: From Data to

830

831

832

833

834

835

836

837

838

839

840

841

842

843

844

845

846

847

848

849

850

851

852

853

854

855

856

857

858

859 Knowledge in 4 Volumes, 231-261.

Sadowski, J., Schwab, C.H., and Gasteiger, J. (2004). 3D structure generation and conformational searching. Dekker Inc., New York.

Sałat, K., Podkowa, A., Malikowska, N., Kern, F., Pabel, J., Wojcieszak, E., Kulig, K., Wanner, K.T., Strach, B., and Wyska, E. (2017). Novel, highly potent and in vivo active inhibitor of GABA transporter subtype 1 with anticonvulsant, anxiolytic, antidepressant and antinociceptive properties. Neuropharmacology 113, 331-342.

Šali, A., Potterton, L., Yuan, F., Van Vlijmen, H., and Karplus, M. (1995). Evaluation of comparative protein modeling by MODELLER. Proteins: Structure, Function, and Bioinformatics 23, 318-326.

Schmidt, S.K., Höfner, G., and Wanner, K.T. (2017). Determination of enantiomeric excess of nipecotic acid as 1-(7-nitrobenzo [c][1, 2, 5] oxadiazol-4-yl) derivatives. Chirality 29, 4856.

Schmuker, M., Givehchi, A., and Schneider, G. (2004). Impact of different software implementations on the performance of the Maxmin method for diverse subset selection. Molecular diversity 8, 421-425.

Schousboe, A. (2000). Pharmacological and functional characterization of astrocytic GABA transport: a short review. Neurochemical research 25, 1241-1244.

Sherin, J.E., and Nemeroff, C.B. (2011). Post-traumatic stress disorder: the neurobiological impact of psychological trauma. Dialogues in clinical neuroscience 13, 263.

Sherman, W., Day, T., Jacobson, M.P., Friesner, R.A., and Farid, R. (2006). Novel procedure for modeling ligand/receptor induced fit effects. Journal of medicinal chemistry 49, 534-553.

Shetty, A.K., and Bates, A. (2015). Potential of GABA-ergic cell therapy for schizophrenia, neuropathic pain, and Alzheimer' s and Parkinson's diseases. Brain research.

Sitka, I., Allmendinger, L., Fülep, G., Höfner, G., and Wanner, K.T. (2013). Synthesis of Nsubstituted acyclic $\beta$-amino acids and their investigation as GABA uptake inhibitors. European journal of medicinal chemistry 65, 487-499.

Skovstrup, S., Taboureau, O., Bräuner-Osborne, H., and Jørgensen, F.S. (2010). Homology modelling of the GABA transporter and analysis of tiagabine binding. ChemMedChem 5, 986-1000. 
860 Stanton, D.T., Morris, T.W., Roychoudhury, S., and Parker, C.N. (1999). Application of nearest-

861

862

863

864

865

866

867

868

869

870

871

872

873

874

875

876

877

878

879

880

881

882

883

884

885

886

887

888

889

890 neighbor and cluster analyses in pharmaceutical lead discovery. Journal of chemical information and computer sciences 39, 21-27.

Tóth, K., Höfner, G., and Wanner, K.T. (2018). Synthesis and biological evaluation of novel Nsubstituted nipecotic acid derivatives with a trans-alkene spacer as potent GABA uptake inhibitors. Bioorganic \& Medicinal Chemistry.

Wang, K.H., Penmatsa, A., and Gouaux, E. (2015a). Neurotransmitter and psychostimulant recognition by the dopamine transporter. Nature.

Wang, K.H., Penmatsa, A., and Gouaux, E. (2015b). Neurotransmitter and psychostimulant recognition by the dopamine transporter. Nature 521, 322-327.

Wermuth, P.B.C.G. (2008). "The Practice of Medicinal Chemistry." Elsevier BV), 319-357.

Yamashita, A., Singh, S., Kawate, T., Jin, Y., and Gouaux, E. (2005). Crystal structure of a bacterial homologue of Na'. Cl (-dependent neurotransmitter transporters. Nature 437: $215 A 223$.

Yu, N., Cao, Y., Mager, S., and Lester, H.A. (1998). Topological localization of cysteine 74 in the GABA transporter, GAT1, and its importance in ion binding and permeation. FEBS letters 426, 174-178.

Zafar, S., and Jabeen, I. (2018). Structure, Function, and Modulation of $\gamma$-Aminobutyric Acid Transporter 1 (GAT1) in Neurological Disorders: A Pharmacoinformatic Prospective. Frontiers in chemistry 6.

Zhao, X., Hoesl, C.E., Hoefner, G.C., and Wanner, K.T. (2005). Synthesis and biological evaluation of new GABA-uptake inhibitors derived from proline and from pyrrolidine-2acetic acid. European journal of medicinal chemistry 40, 231-247.

Zheng, J., Wen, R., Luo, X., Lin, G., Zhang, J., Xu, L., Guo, L., and Jiang, H. (2006). Design, synthesis, and biological evaluation of the N-diarylalkenyl-piperidinecarboxylic acid derivatives as GABA uptake inhibitors (I). Bioorganic \& medicinal chemistry letters 16 , 225-227.

Zhou, Y., Zomot, E., and Kanner, B.I. (2006). Identification of a lithium interaction site in the $\gamma$ aminobutyric acid (GABA) transporter GAT-1. Journal of Biological Chemistry 281, 22092-22099. 


\section{Figure 1}

Workflow of the hGAT1 antagonists data pre-processing and cleaning
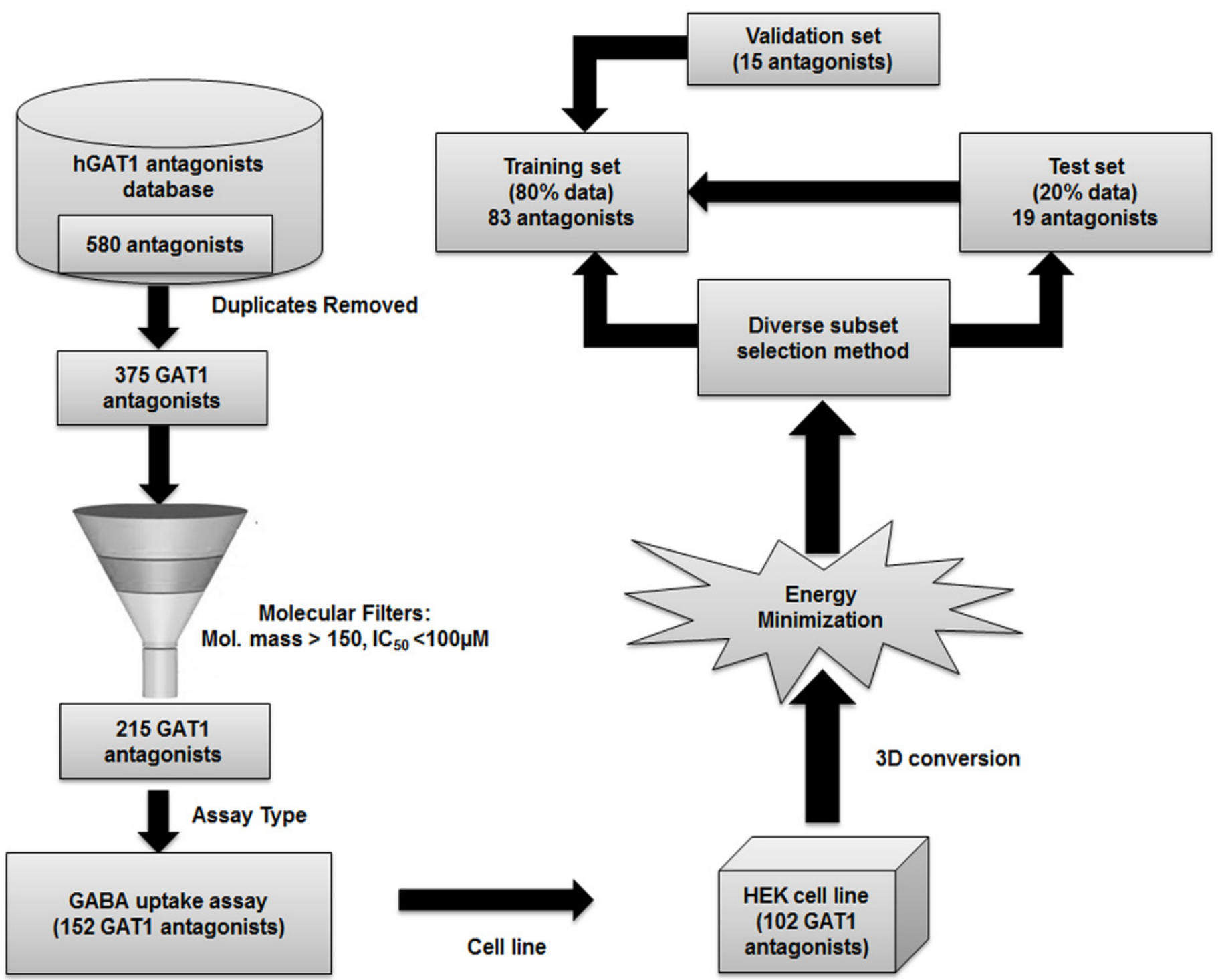
Figure 2

Best aligned conformations of complete data set (102 compounds) of hGAT1 antagonists on standard 3D conformation of Tiagabine as a template.

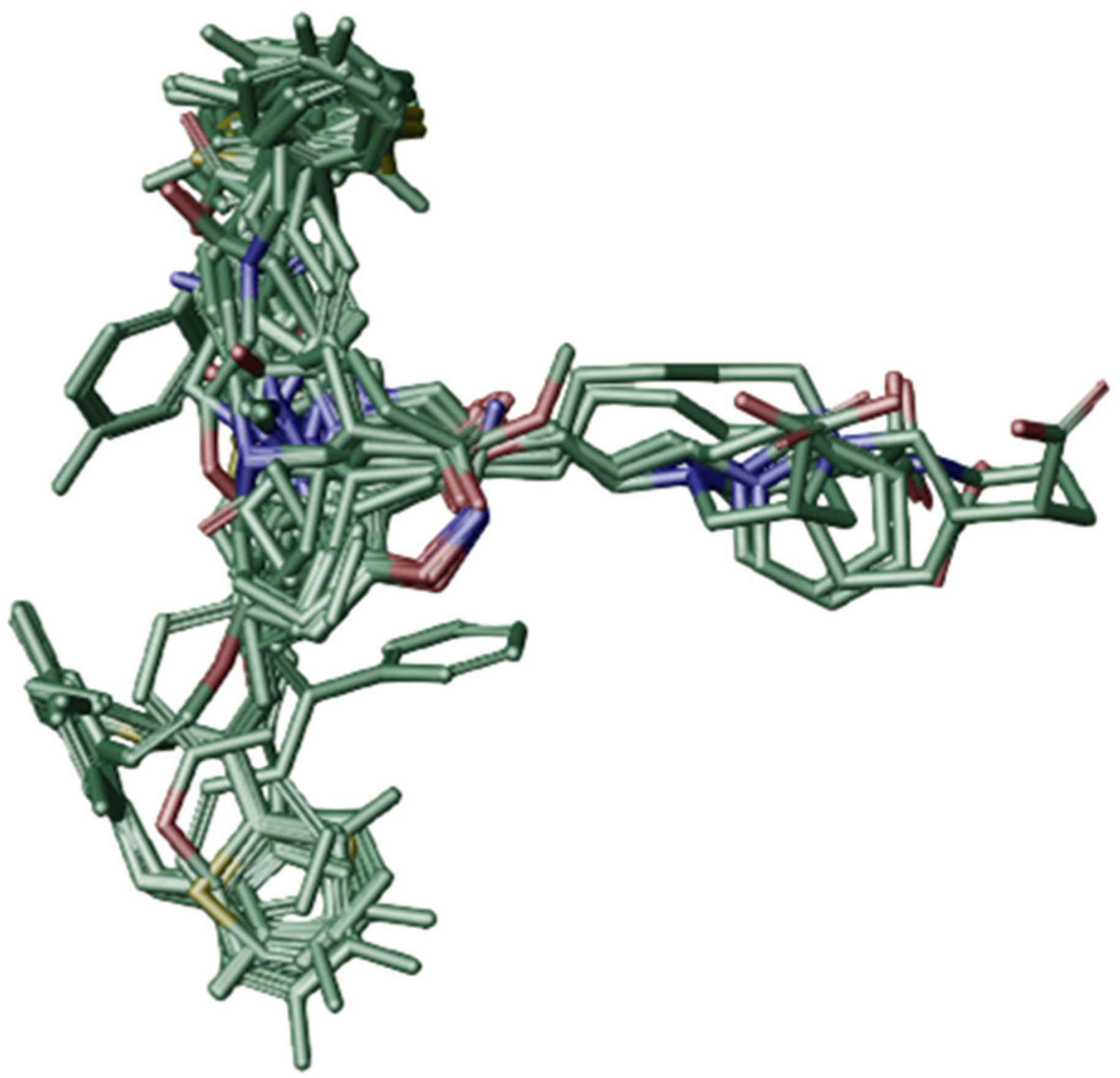


Figure 3

Correlation plot between experimental versus predicted inhibitory potencies $\left(-\log I C_{50}\right)$ of hGAT1 antagonists.

Training set, test set and validation set are represented with filled circles, hollow circles and triangles, respectively. The chemical structure represents observed outlier (compound 35) in the training set.

*Note: Auto Gamma Correction was used for the image. This only affects the reviewing manuscript. See original source image if needed for review.

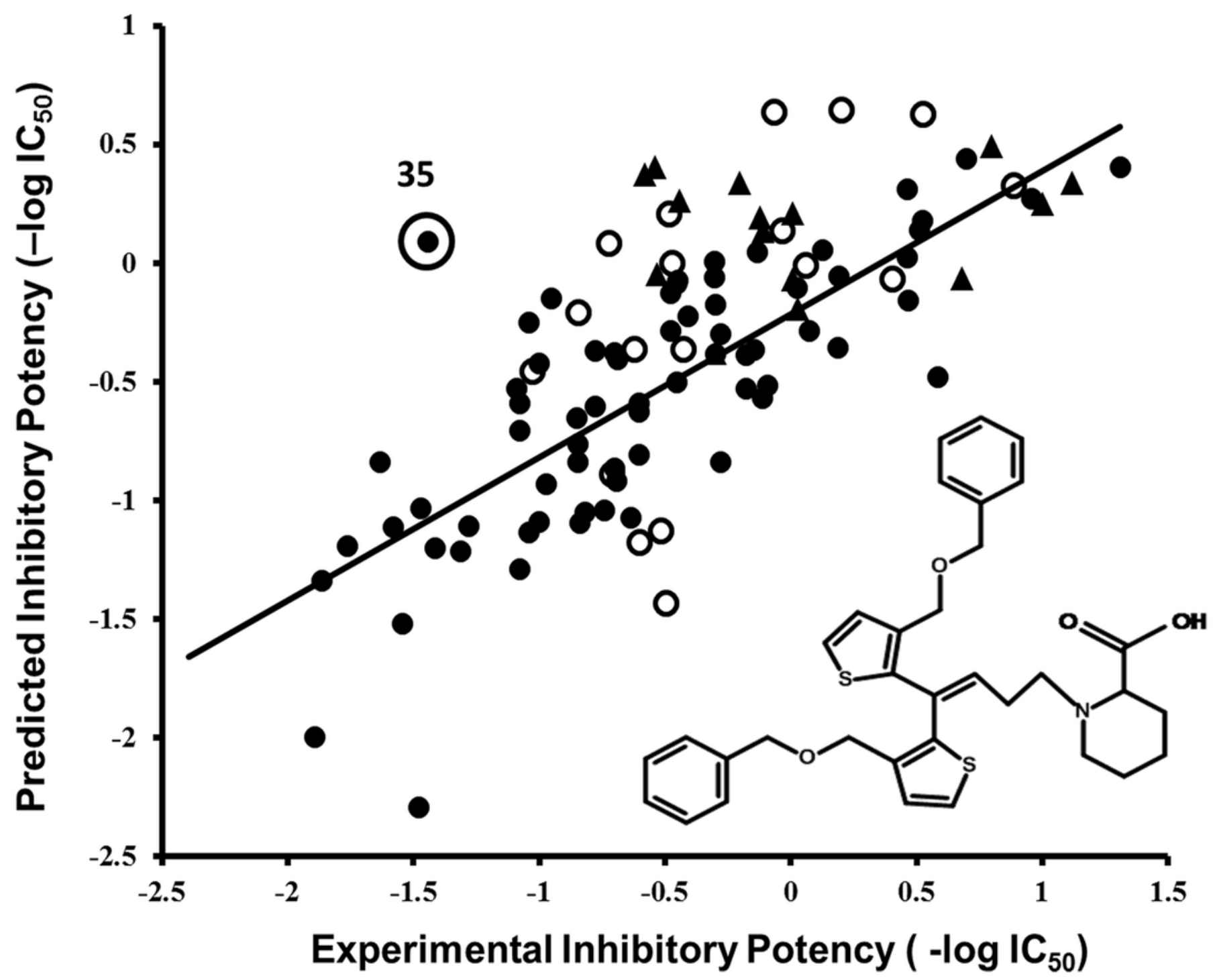




\section{Figure 4}

PLS coefficients correlograms showing the descriptors directly (positive value) or inversely (negative values) correlated to $-\log \mathrm{IC}_{50}$ values of the dataset.

A change in biological activity against hGAT1 is depicted by N1-N1, O-N1, DRY-N1, TIP-TIP and O-O variables. (A) N1-N1 probes (blue contours) represents two hydrogen bond acceptor

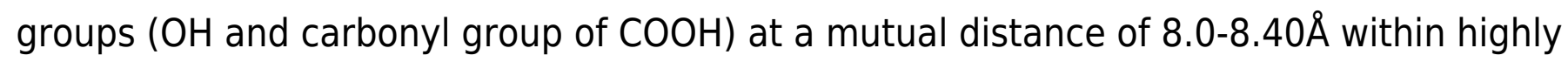
active ligands $(0.049-0.75 \mu \mathrm{M})$ whereas $\mathbf{B}$ ) represent N1-N1 pair of probes at a distance of

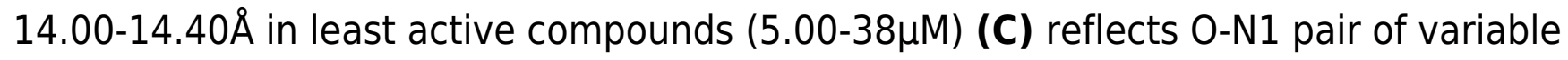
depicting a hydrogen bond donor (O: red contour) and a hydrogen bond acceptor (N1: blue

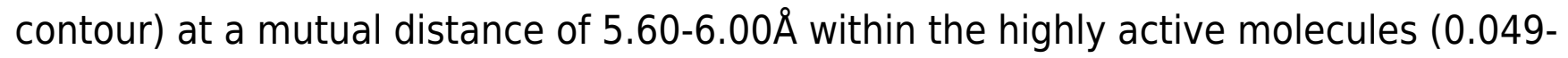

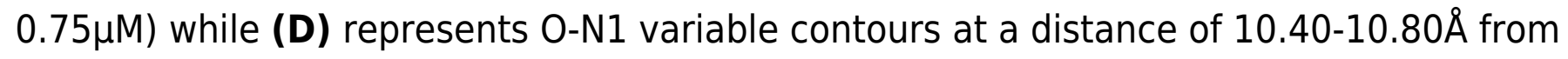

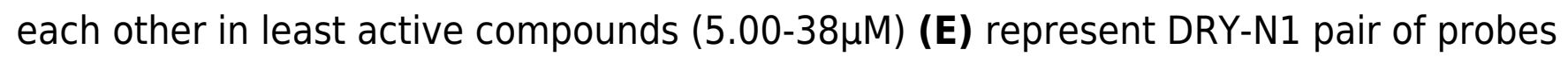

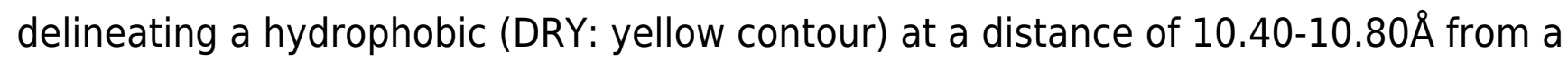
hydrogen bond acceptor region (N1: blue contour) in the active ligands $(0.049-0.75 \mu \mathrm{M})$ (F) represent DRY-N1 pair of probe at distance of $6.40-6.80 \AA$ in least active compounds (4.34$78 \mu \mathrm{M})(\mathbf{G}, \mathbf{H})$ represent $0-0$ variables depicting two hydrogen bond donor probes (O: red contours) at a mutual distance of $6.00-6.40 \AA$ within the molecules; (I) TIP-TIP pair of variable

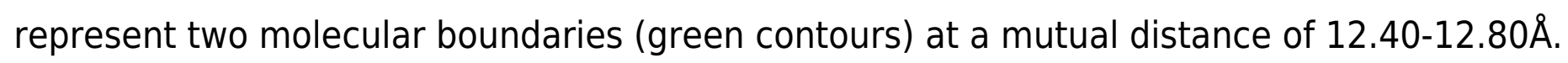




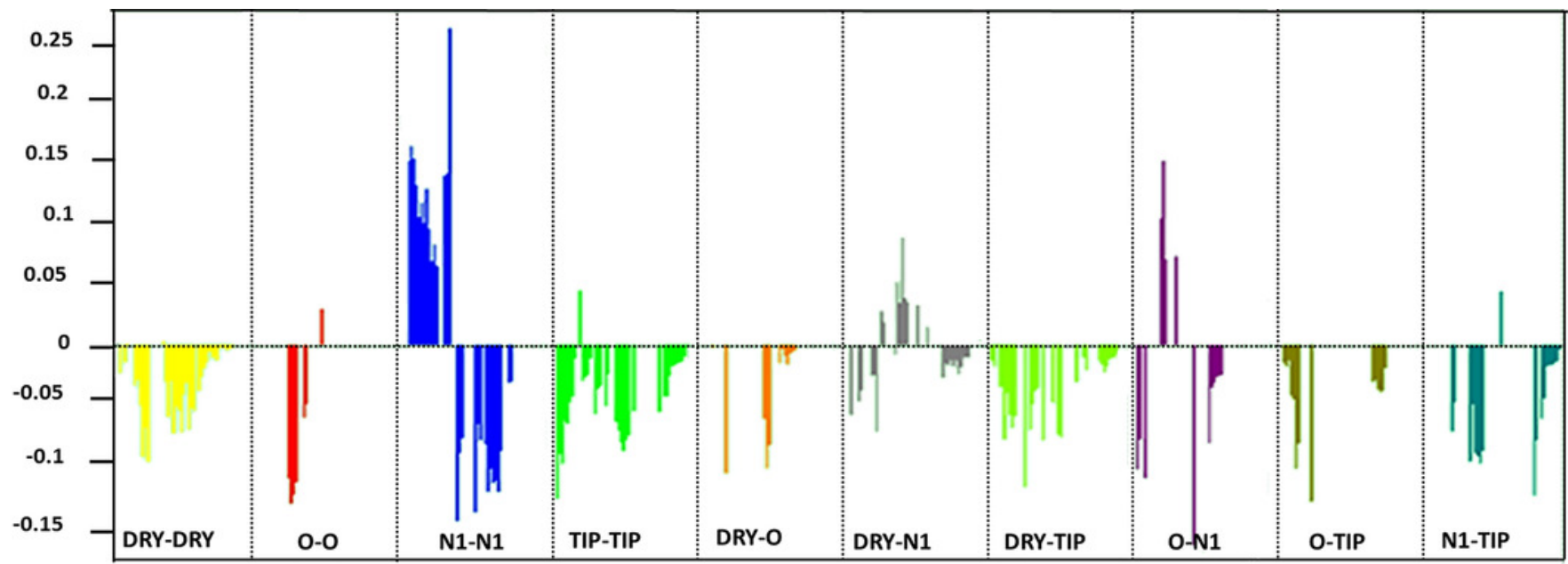

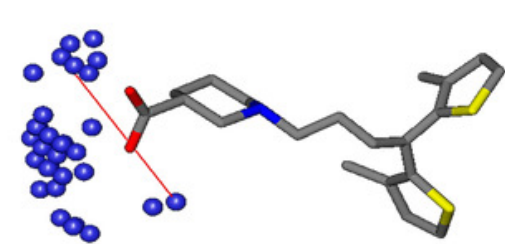

(A) N1-N1 Hotspots

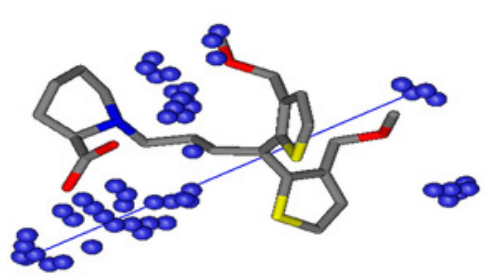

(B) N1-N1 Hotspots

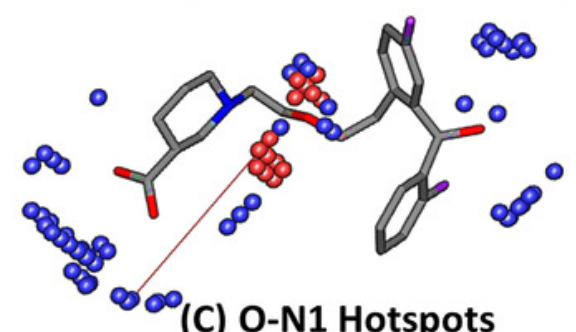

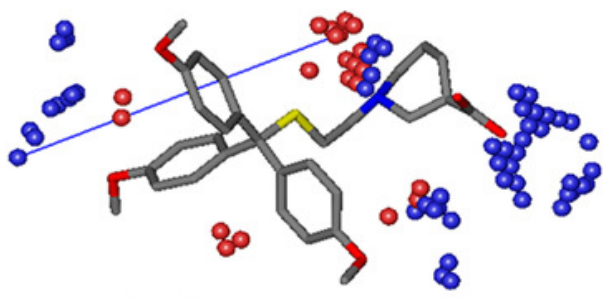

(D) O-N1 Hotspots

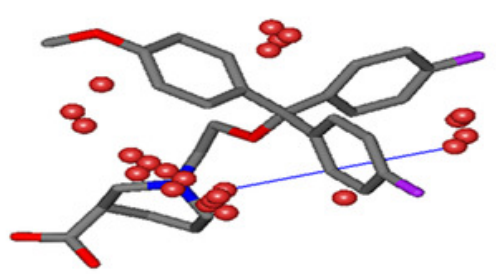

(G) 0-O Hotspots

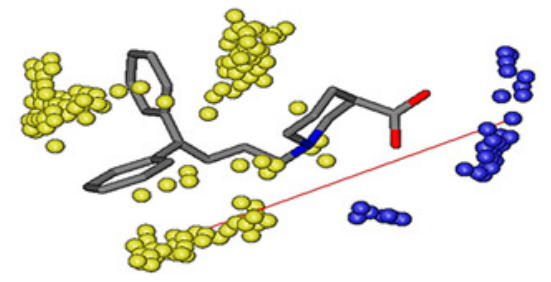

(E) DRY-N1 Hotspots

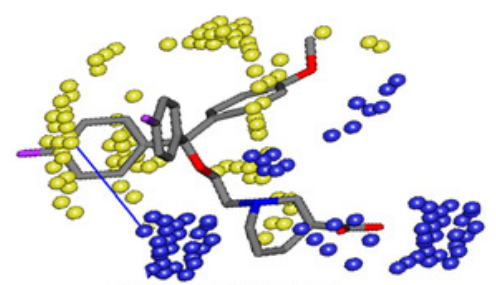

(F) DRY-N1 Hotspots

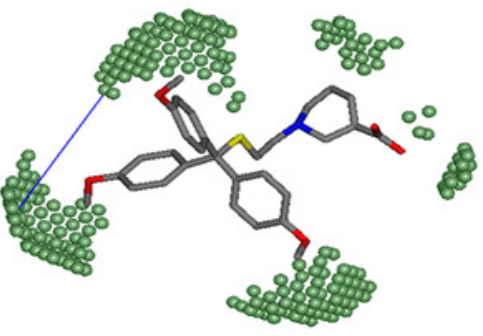

(I) TIP-TIP Hotspots

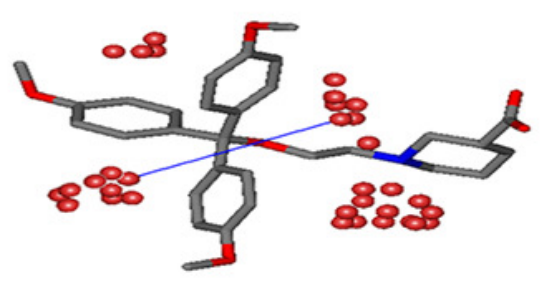

(H) O-O Hotspots 
Figure 5

Docking poses of clusters from class A, B and C in hGAT1 binding pocket that contained maximum number of docked ligands from respective classes.

(A) 14 clusters from class A containing $3(\mathbf{2}, \mathbf{8}, \mathbf{1 5})$ out of 4 ligands. (B) 12 clusters from class B exhibiting $3(\mathbf{1 4}, \mathbf{2 7}, \mathbf{3 6})$ out of 4 ligands. (C) 4 clusters from class C possessing $4(\mathbf{1}$, $\mathbf{3 , 3 7 , 8 4 )}$ out of 5 ligands.
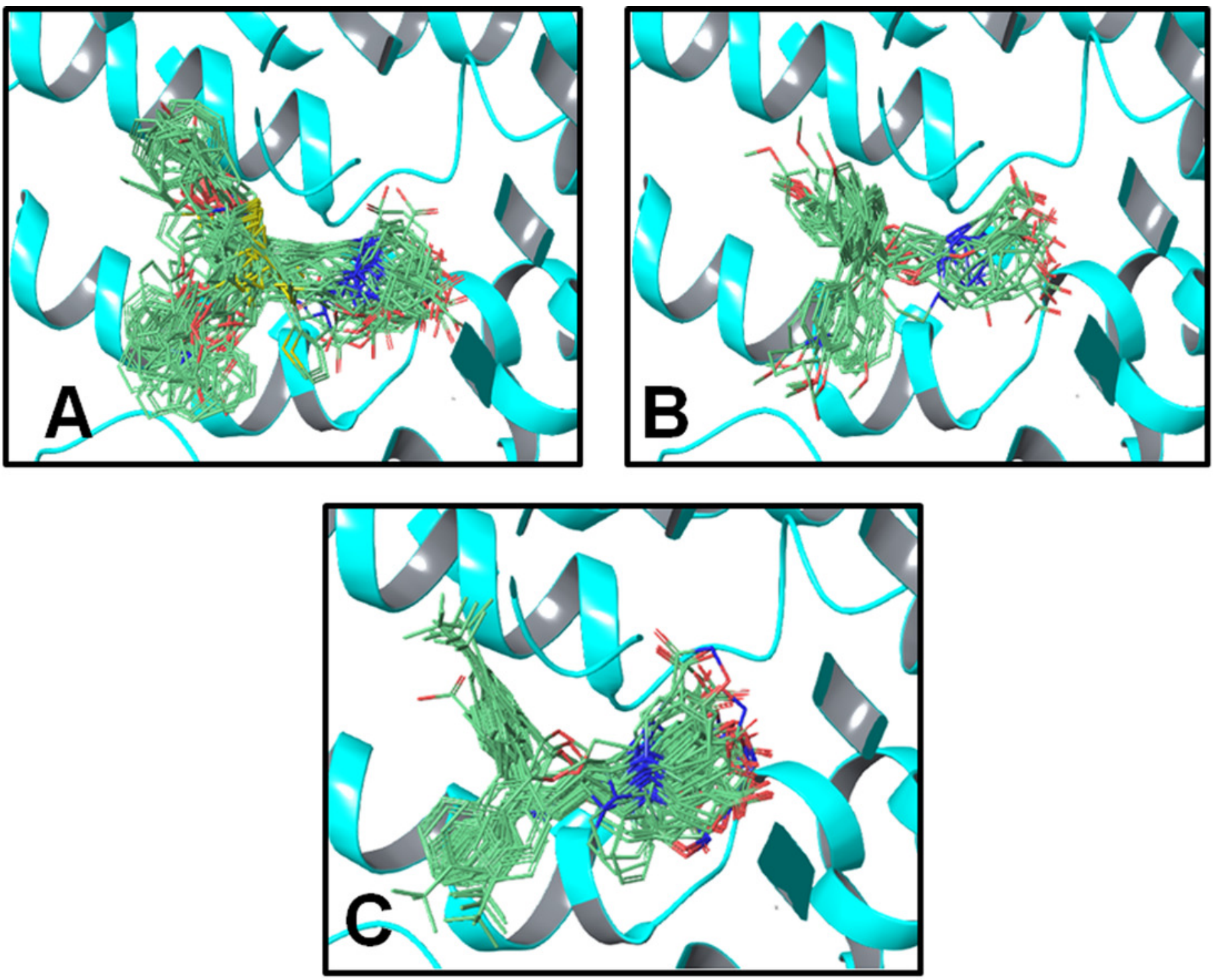


\section{Figure 6}

Winning clusters obtained from docking of hGAT1 antagonists.

(A) Final docking poses of hGAT1 antagonists from class A, B and C. Positioning of di- or triaromatic rings represent the hydrophobic areas and charged piperidine ring represent the polar region in the binding pocket of hGAT1. (B) Binding positions of winning clusters of class $\mathrm{A}, \mathrm{B}$ and $\mathrm{C}$ in hGAT1 binding pocket along with $\mathrm{Na1}, \mathrm{Na2}$ (blue spheres) and a $\mathrm{Cl}$ (green sphere) ion. 


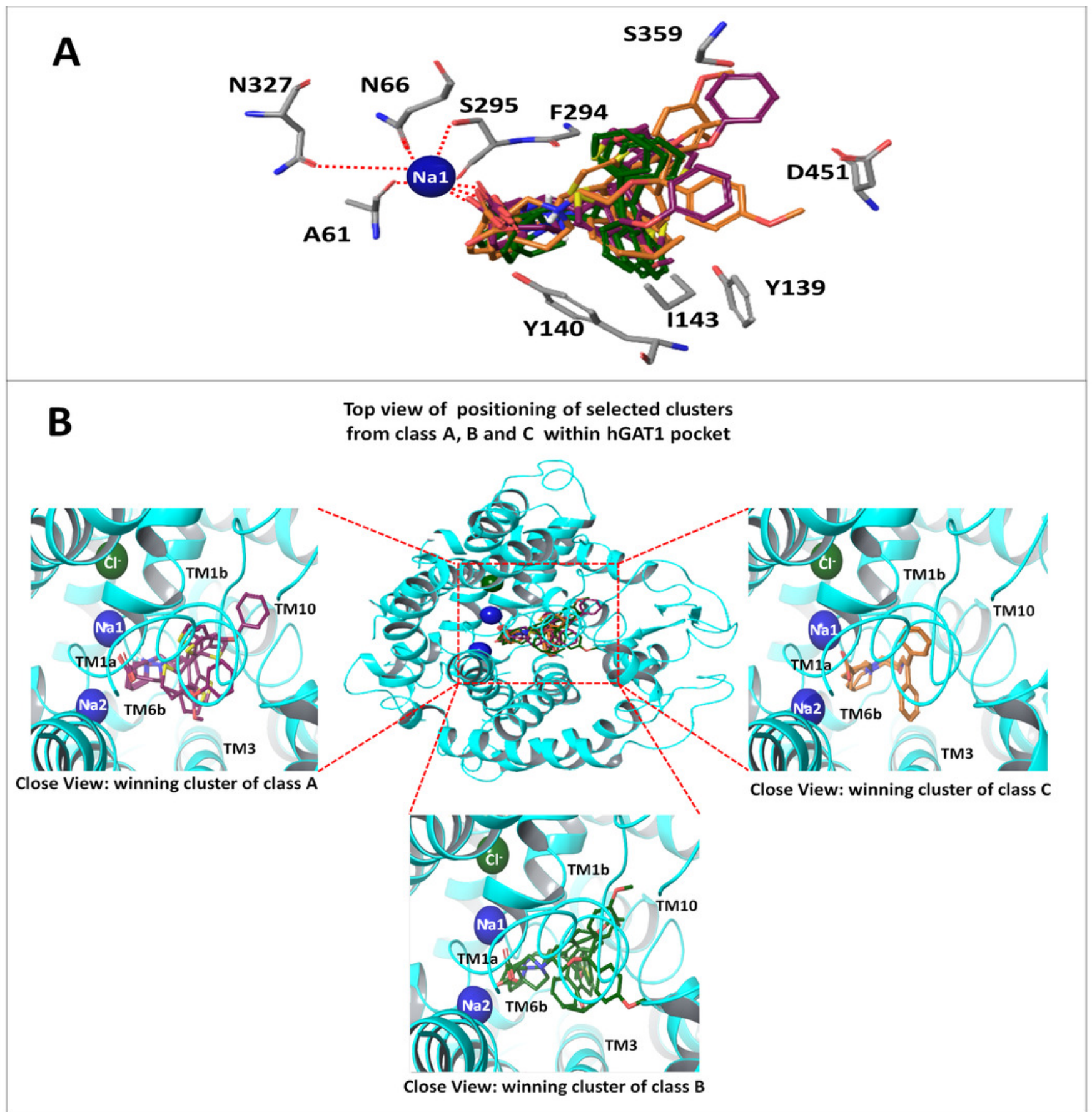


Figure 7

Interaction pattern of binding solutions of class A, B and C in hGAT1 model.

Ligand protein interactions of (A) compound $\mathbf{2}$ and (B) compound $\mathbf{8}$ of class A. Interactions pattern of (C) compound $\mathbf{1 4}$ and (D) compound $\mathbf{3 6}$ of class B. Binding poses of (E) compound $\mathbf{1},(\mathbf{F})$ compound $\mathbf{3}$ and (G) compound $\mathbf{3 7}$ of class $\mathrm{C}$.

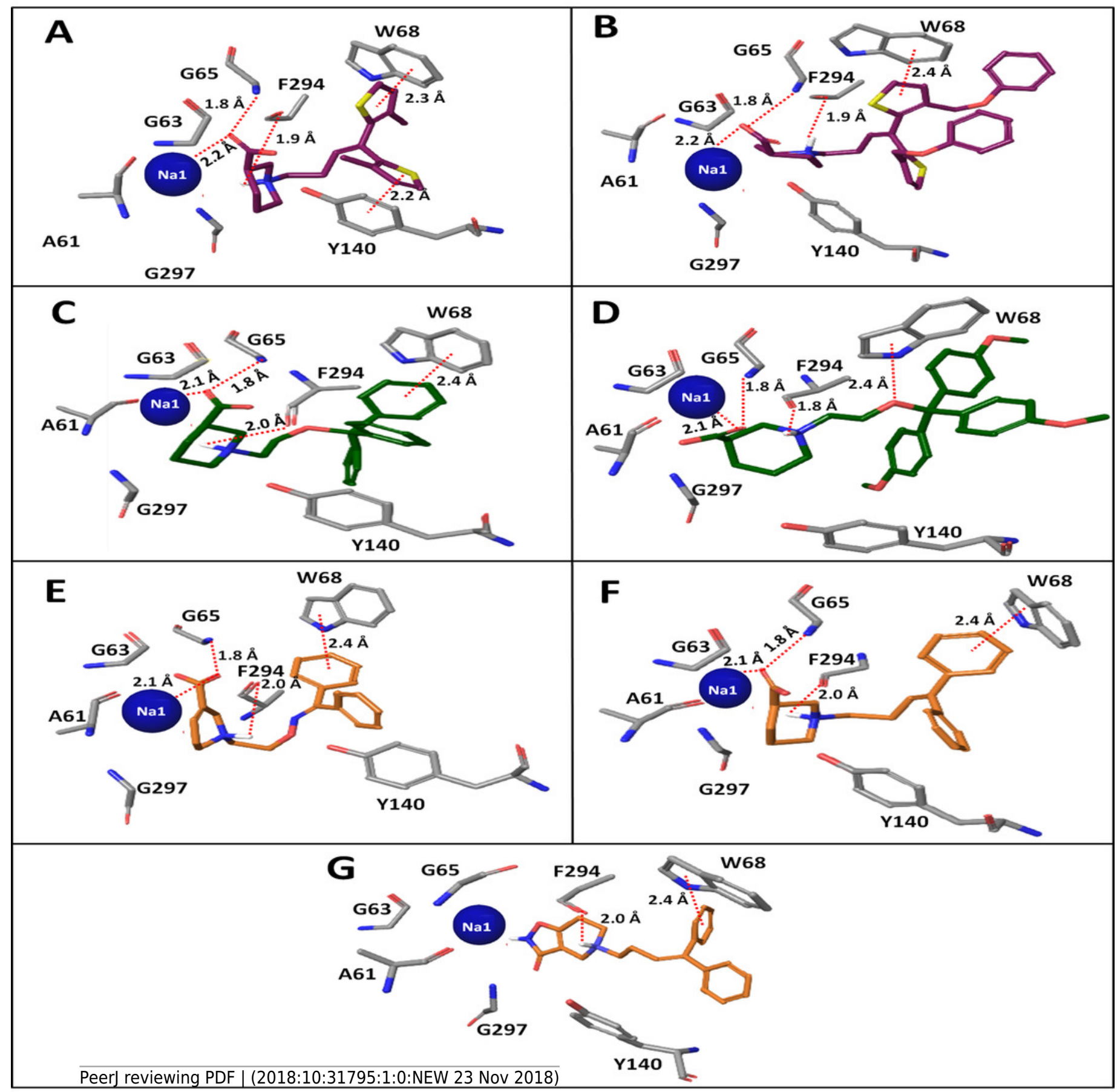




\section{Figure 8}

Outward projection of bulky substitutions on aromatic moieties of antagonists of class A and $B$ from the hGAT1 binding pocket

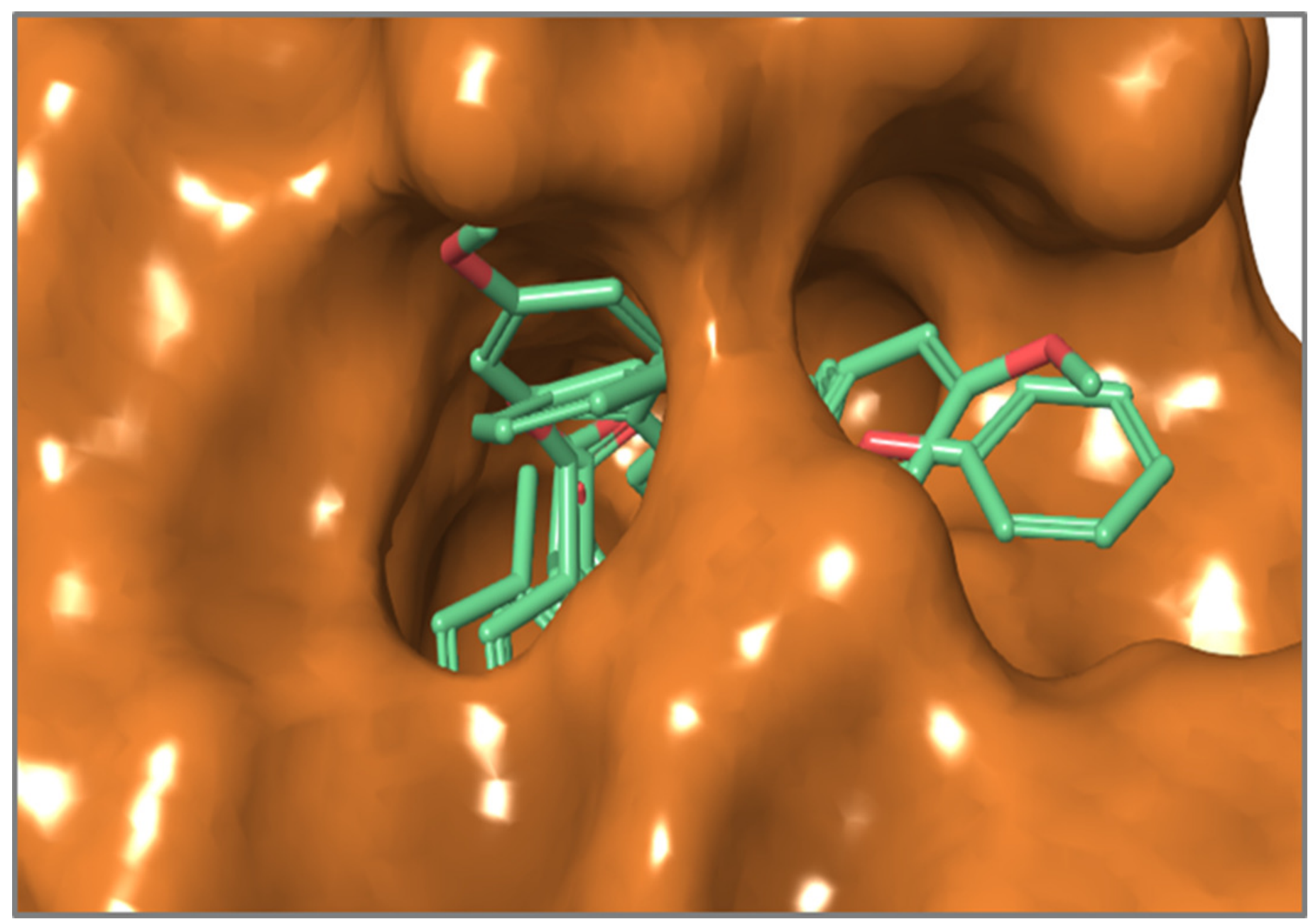




\section{Figure 9}

Important hotspots regions for the high inhibitory potency of hGAT1 inhibitors

One hydrogen bond acceptor contour (N1) at a distance of 8.00-8.40 $\AA$ from second hydrogen bond acceptor (N1) group, at a distance of 5.60-6.00 ^ from a hydrogen bond donor (O) and at a distance of $10.40-10.80 \AA$ from a hydrophobic (DRY) group

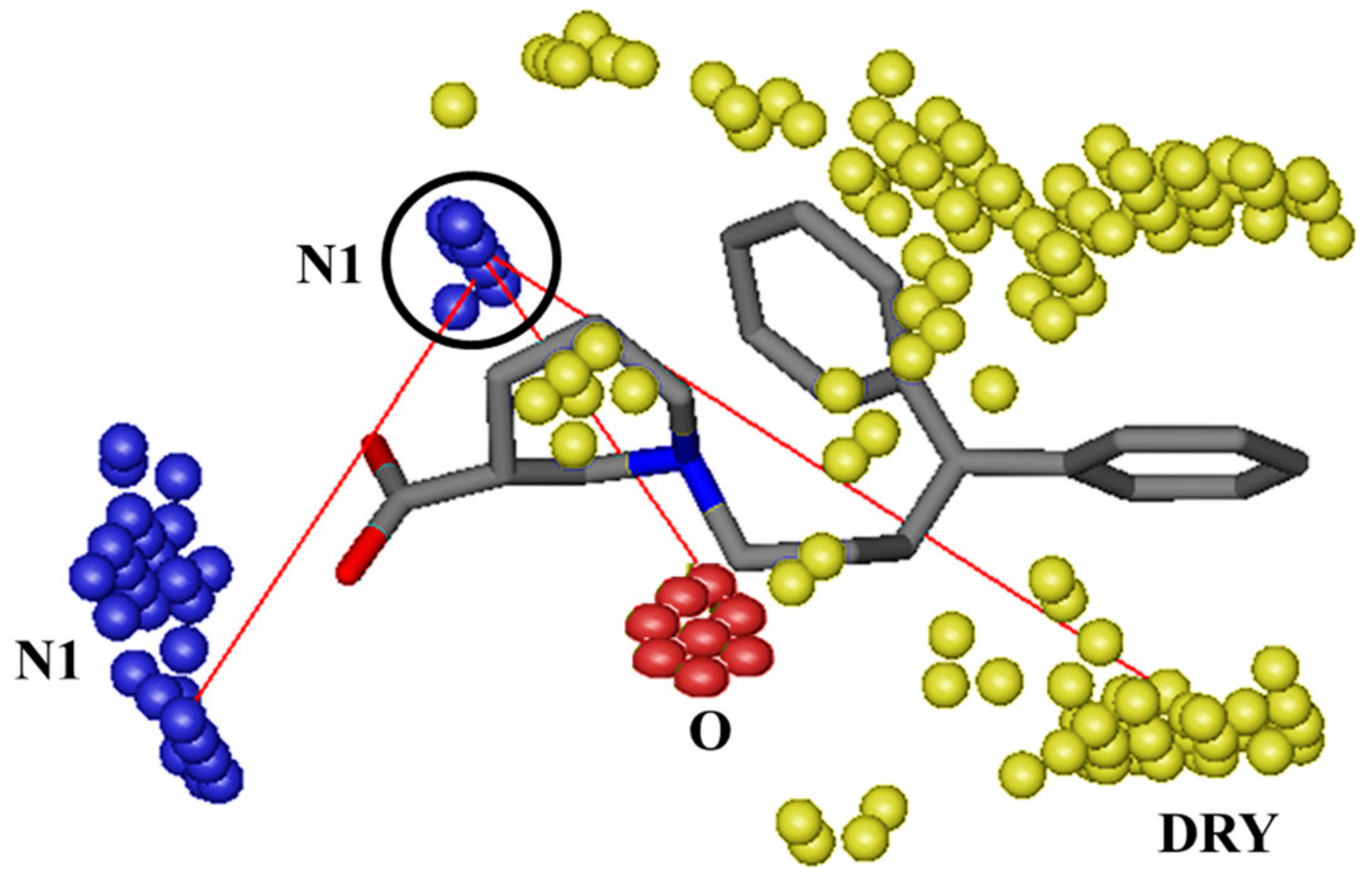




\section{Table $\mathbf{1}$ (on next page)}

Summary of GRIND variables and their corresponding distances identified as highly correlated to biological activity $\left(-\log \mid C_{50}\right)$ of compounds. 


\begin{tabular}{|c|c|c|c|c|}
\hline Probes & $\begin{array}{l}\text { Distances } \\
\text { (Å) }\end{array}$ & Features & Impact & Comments \\
\hline \multirow[b]{2}{*}{ N1-N1 } & $8.00-8.40$ & $\begin{array}{c}\mathrm{OH} \\
\mathrm{C}=\mathrm{O}\end{array}$ & + & $\begin{array}{l}\mathrm{COOH} \text { group at meta position of } \\
\text { piperidine, pyrrolidine, or azetidine ring } \\
\text { has shown positive contribution towards } \\
\text { hGAT1 inhibition activity }\left(\mathrm{IC}_{50}\right)\end{array}$ \\
\hline & $14.00-14.40$ & $\begin{array}{l}\mathrm{C}=\mathrm{O} \\
-\mathrm{O}-\end{array}$ & - & $\begin{array}{l}\text { Carbonyl oxygen of the } \mathrm{COOH} \text { group } \\
\text { and the ether group present either in the } \\
\text { linker region or in the bulky aromatic } \\
\text { substituents has shown negative } \\
\text { contribution towards the biological } \\
\text { activity. }\end{array}$ \\
\hline \multirow{2}{*}{ O-N1 } & $5.60-6.00$ & $\stackrel{\mathrm{COOH}}{-\mathrm{N}}^{+-}$ & + & $\begin{array}{l}\text { Distance between protonated nitrogen of } \\
\text { the piperidine, pyrrolidine, or azetidine } \\
\text { ring and the } \mathrm{COOH} \text { group }\end{array}$ \\
\hline & $10.40-10.80$ & $\stackrel{-\mathrm{N}^{+-}}{\mathrm{OCH}_{3}}$ & - & $\begin{array}{l}\text { Protonated nitrogen of the piperidine ring } \\
\text { and the methoxy substitution of the diaryl } \\
\text { moieties }\end{array}$ \\
\hline \multirow[t]{2}{*}{ DRY-N1 } & $10.40-10.80$ & $\begin{array}{l}\text { Di/tri aryl } \\
\text { moieties } \\
\mathrm{COOH}\end{array}$ & + & $\begin{array}{l}\text { Distance between } \mathrm{COOH} \text { group of } \\
\text { piperidine, proline, pyrrolidine, or } \\
\text { azetidine ring and bulky aromatic rings } \\
\text { after linker chain }\end{array}$ \\
\hline & $6.40-6.80$ & $\begin{array}{l}\text { Di/tri aryl } \\
\text { moieties } \\
\text {-O- }\end{array}$ & - & $\begin{array}{l}\text { Distance between aromatic moieties and } \\
\text { the ether group in the linker region }\end{array}$ \\
\hline $\mathrm{O}-\mathrm{O}$ & $6.00-6.40$ & $\begin{array}{l}-\mathrm{O}- \\
\mathrm{X} \text { atom (any } \\
\text { electronegative } \\
\text { atom e.g., } \mathrm{F}, \mathrm{Cl}^{-} \text {, } \\
\left.\mathrm{O}^{-}, \mathrm{F}^{-}\right)\end{array}$ & - & $\begin{array}{l}\text { Depicts a distance between the ether } \\
\text { group of hydrophilic chain and methoxy } \\
\text { or flouro group attached at para position } \\
\text { of aromatic rings }\end{array}$ \\
\hline TIP-TIP & $12.40-12.80$ & $\mathrm{OCH}_{3}$ & - & the methoxy \\
\hline
\end{tabular}




\begin{tabular}{|l|l|l|l|}
\hline & \multicolumn{2}{|l|}{} & $\begin{array}{l}\text { substitutions on aromatic moieties } \\
\text { attached at the linker region of hGAT1 } \\
\text { antagonists }\end{array}$ \\
\hline
\end{tabular}

1 
Table 2 (on next page)

External validation set of nipecotic acid derivatives of hGAT1 inhibitors. 


\begin{tabular}{|c|c|c|c|c|c|c|c|}
\hline $\begin{array}{c}\text { Validation } \\
\text { Set } \\
\text { Compound } \\
\text { \# (VSC) }\end{array}$ & $\mathbf{n}$ & $\mathbf{R}_{1}$ & $\mathbf{R}_{2}$ & $\mathrm{IC}_{50} \boldsymbol{\mu M}$ & $-\log \mathrm{IC}_{50}$ & $\begin{array}{c}\text { Predicted } \\
-\log \mathrm{IC}_{50}\end{array}$ & $\begin{array}{c}\text { Residual } \\
\text { value }\end{array}$ \\
\hline VSC_1 & 1 & $\mathrm{H}$ & $\mathrm{H}$ & 0.10 & 1.00 & 0.24 & 0.75 \\
\hline $\mathrm{VSC} \_2$ & 1 & $\mathrm{~F}$ & $\mathrm{H}$ & 0.07 & 1.12 & 0.33 & 0.78 \\
\hline VSC_3 & 1 & $\mathrm{CH}_{3}$ & $\mathrm{CH}_{3}$ & 0.20 & 0.68 & -0.06 & 0.74 \\
\hline VSC_4 & 1 & $\mathrm{CH}_{3}$ & $\mathrm{Cl}$ & 0.15 & 0.80 & 0.49 & 0.30 \\
\hline VSC_5 & 2 & $\mathrm{Cl}$ & $\mathrm{H}$ & 1.58 & -0.20 & 0.33 & -0.53 \\
\hline VSC_6 & 2 & $\mathrm{~F}$ & $\mathrm{H}$ & 2.75 & -0.44 & 0.26 & -0.70 \\
\hline VSC_7 & 2 & $\mathrm{CH}_{3}$ & $\mathrm{H}$ & 1.28 & -0.11 & 0.13 & -0.24 \\
\hline VSC_8 & 2 & $\mathrm{H}$ & $\mathrm{Cl}$ & 3.38 & -0.53 & -0.04 & -0.48 \\
\hline VSC_9 & 2 & $\mathrm{Cl}$ & $\mathrm{Cl}$ & 0.93 & 0.03 & -0.19 & 0.22 \\
\hline $\mathrm{VSC}_{-} 10$ & 2 & $\mathrm{~F}$ & $\mathrm{~F}$ & 1.99 & -0.30 & -0.38 & 0.08 \\
\hline VSC_11 & 2 & $\mathrm{Cl}$ & $\mathrm{F}$ & 0.97 & 0.01 & -0.06 & 0.07 \\
\hline VSC_12 & 2 & $\mathrm{CH}_{3}$ & $\mathrm{Cl}$ & 0.97 & 0.01 & 0.20 & -0.19 \\
\hline VSC_13 & 2 & $\mathrm{CH}_{3}$ & $\mathrm{~F}$ & 1.31 & -0.12 & 0.19 & -0.31 \\
\hline
\end{tabular}




\section{Table 3(on next page)}

Selected compounds from class A, B and C for experimental guided docking studies. 
<smiles>[R2]c1ccsc1C(=CCCN1CCCCC1)c1sccc1[R1]</smiles>

Class A

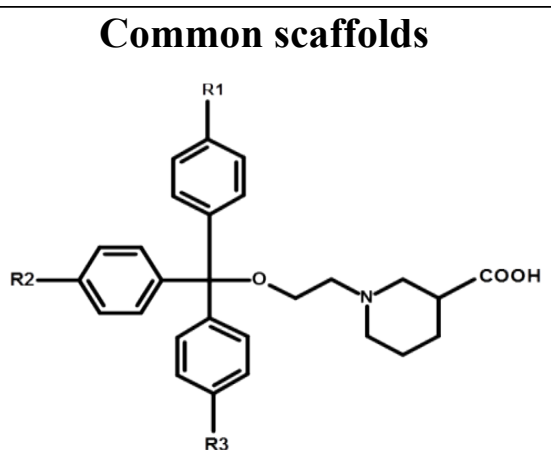

Class B<smiles>[R]CCN1CCC=C(C(=O)O)C1</smiles>

Class C

\begin{tabular}{|c|c|c|c|c|c|}
\hline Compound \# & Class scaffold & $\mathbf{R}_{1}$ & $\mathbf{R}_{\mathbf{2}}$ & $\mathbf{R}_{\mathbf{3}}$ & $I C_{50}(\mu \mathrm{M})$ \\
\hline 2 & $A$ & $-\mathrm{CH}_{3}$ & $-\mathrm{CH}_{3}$ & $\mathrm{~m}-\mathrm{COOH}(\mathrm{R})$ & 0.049 \\
\hline 6 & $A$ & $-\mathrm{CH}_{2} \mathrm{OC}_{6} \mathrm{H}_{5}$ & $-\mathrm{CH}_{2} \mathrm{OC}_{6} \mathrm{H}_{5}$ & $\mathrm{~m}-\mathrm{COOH}(\mathrm{R})$ & 0.34 \\
\hline 8 & $A$ & $-\mathrm{CH}_{2} \mathrm{OC}_{6} \mathrm{H}_{5}$ & $-\mathrm{CH}_{2} \mathrm{OC}_{6} \mathrm{H}_{5}$ & $\mathrm{O}-\mathrm{COOH}(\mathrm{R}, \mathrm{S})$ & 0.65 \\
\hline 15 & A & $-\mathrm{CH}_{2} \mathrm{OC}_{6} \mathrm{H}_{5}$ & $-\mathrm{CH}_{2} \mathrm{OC}_{6} \mathrm{H}_{5}$ & $p-\mathrm{COOH}(\mathrm{R}, \mathrm{S})$ & 1.5 \\
\hline 14 & B & $\mathrm{H}$ & $\mathrm{H}$ & $\mathrm{H}$ & 1.4 \\
\hline 27 & B & $\mathrm{H}$ & $-\mathrm{OCH}_{3}$ & $\mathrm{H}$ & 6.9 \\
\hline 36 & B & $-\mathrm{OCH}_{3}$ & $-\mathrm{OCH}_{3}$ & $-\mathrm{OCH}_{3}$ & 30 \\
\hline 40 & $B$ & $-\mathrm{OCH}_{3}$ & $-\mathrm{OCH}_{3}$ & $\mathrm{H}$ & 43 \\
\hline 1 & C & $-\mathrm{ON}=\mathrm{C}\left(\mathrm{C}_{6} \mathrm{H}_{5}\right)_{2}$ & - & - & 0.040 \\
\hline 3 & C & $-\mathrm{CH}=\mathrm{C}\left(\mathrm{C}_{6} \mathrm{H}_{5}\right)_{2}$ & - & - & 0.20 \\
\hline 4 & $\mathrm{C}$ & $-\mathrm{OCH}\left(\mathrm{C}_{6} \mathrm{H}_{5}-\mathrm{m}-\mathrm{CF}_{3}\right)_{2}$ & - & - & 0.26 \\
\hline 37 & C & & & & 38 \\
\hline 84 & $\mathrm{C}$ & & & & 0.11 \\
\hline
\end{tabular}

